\title{
CINCO DÉCADAS DE PROGRAMAS EUROPEOS SOBRE MEDIO AMBIENTE. UN REPASO ANTE LA LLEGADA DEL OCTAVO PROGRAMA
}

\section{FIVE DECADES OF EUROPEAN ENVIRONMENTAL PROGRAMS. A REVIEW BEFORE THE ARRIVAL OF THE EIGHTH PROGRAM}

\author{
Asier GarcíA LUPIOLA \\ Profesor Agregado / Investigador en formación \\ UPV-EHU / UNED \\ $\underline{\text { asier.garcialupiola@ehu.eus }}$
}

Fecha de recepción: 21 de julio de 2021 / Fecha de aceptación: 15 de noviembre de 2021

RESUMEN: La política medioambiental de la UE tiene su origen en las primeras decisiones adoptadas a comienzos de la década de 1970. Desde entonces, los programas de acción sobre medio ambiente han concretado los fundamentos y objetivos generales en la materia. Inicialmente, a ellos les correspondió definir las bases de la política medioambiental y a partir del AUE, constituyen la herramienta para fijar el desarrollo de la normativa fundamental recogida en los Tratados. De este modo, determinan el camino que deben seguir los actos legislativos que conforman la política medioambiental europea, así como todas las demás políticas en lo que se refiere a la exigencia de protección medioambiental que deben integrar todas ellas. En estos cincuenta años, los programas de acción sobre medio ambiente se han convertido en un gran exponente del objetivo de desarrollo sostenible de la UE.

RESUM: La política mediambiental de la UE té el seu origen en les primeres decisions adoptades al començament de la dècada de 1970. Des de llavors, els programes d'acció sobre medi ambient han concretat els fonaments i objectius generals en la matèria. Inicialment, a ells els va correspondre definir les bases 
de la política mediambiental i a partir de l'AUE, constitueixen l'eina per fixar el desenvolupament de la normativa fonamental recollida en els Tractats. D'aquesta manera, determinen el camí que han de seguir els actes legislatius que conformen la política mediambiental europea, així com totes les altres polítiques pel que fa a l'exigència de protecció mediambiental que han d'integrar totes elles. En aquests cinquanta anys, els programes d'acció sobre medi ambient s'han convertit en un gran exponent de l'objectiu de desenvolupament sostenible de la UE.

ABSTRACT: The EU's environmental policy has its origin in the first decisions taken in the early 1970s. Since then, the environmental action programs have specified the fundamentals and general objectives in this area. Initially, they defined the bases of environmental policy and, since the European Single Act, they constitute the tool to establish the development of the fundamental regulations contained in the Treaties. They determine the path to be followed by the legislative acts that make up the European environmental policy, as well as all other policies that must integrate the requirement of environmental protection. In these fifty years, the action programs on the environment have become a great exponent of the EU's sustainable development objective.

PALABRAS CLAVE: Unión Europea - Política medioambiental - Programa de acción - Desarrollo sostenible

PARAULES CLAU: Unió Europea - Política mediambiental - Programa d'acció - Desenvolupament sostenible

KEY WORDS: European Union - Environmental Policy - Action Program Sustainable Development

SUMARIO: I. INTRODUCCIÓN. II. ANTECEDENTES. III. LOS PRIMEROS PROGRAMAS DE ACCIÓN SOBRE MEDIO AMBIENTE. IV. EL ACTA ÚNICA EUROPEA Y EL CUARTO PMA. V. EL TRATADO DE MAASTRICHT Y EL QUINTO PMA. VI. EL TRATADO DE ÁMSTERDAM Y EL SEXTO PMA. VII. EL TRATADO DE LISBOA Y EL SÉPTIMO PMA. VIII. LA NECESIDAD DE UNA MAYOR AMBICIÓN. IX. EL PACTO VERDE EUROPEO Y EL OCTAVO PMA. X. CONCLUSIONES.

\section{INTRODUCCIÓN}


El medio ambiente constituye una de las mayores preocupaciones de la sociedad, que demanda una adecuada protección del mismo, más aún ante la relevancia de los efectos del cambio climático. La variedad y gravedad de las consecuencias derivadas de la falta de respeto por el medio ambiente y la necesidad de conjugar la defensa ambiental con el crecimiento económico convierten el desarrollo sostenible en uno de los retos principales en el plano internacional. La normativa vigente en la Unión Europea (UE) determina que uno de los objetivos fundamentales del proceso de integración es, precisamente, el desarrollo sostenible (art. 3 del Tratado de la UE, TUE).

Lo cierto es que los Tratados constitutivos de las Comunidades Europeas (CCEE) no establecieron dicho objetivo, si bien gracias a las diferentes reformas habidas en los mismos acabó recogido en los textos jurídicos fundamentales de la UE -hoy día, además del TUE, el Tratado sobre el Funcionamiento de la UE (TFUE)-. De esta manera, el desarrollo sostenible constituye un fin vinculante para todos los Estados miembros de la UE y para el cumplimiento de dicho objetivo el desarrollo de la política europea de medio ambiente ha sido fundamental. Una de las herramientas básicas para ir diseñando dicha política ha sido el conjunto de Programas de Acción sobre Medio Ambiente (PMA) que las instituciones europeas han ido adoptando desde la década de 1970.

A ellos se recurrió incluso antes de incorporar a los Tratados el medio ambiente como competencia de las CCEE, algo que sucedió con el Acta Única Europea (AUE) de 1986. Ahora bien, se trata de una competencia compartida entre la UE y los Estados miembros (art. 4.2 TFUE), que faculta a la UE a actuar y legislar de manera que si lo hace, los Estados miembros no podrán hacerlo (art. 2.2 TFUE). En la materia que nos ocupa, la UE ha utilizado activamente el poder que le otorga el Tratado, teniendo presente siempre las directrices a seguir para alcanzar los objetivos prioritarios, que han de ser fijados, precisamente, por los programas de acción europeos (art. 192.3 TFUE). Como se ve, la relevancia de los PMA radica en que constituyen el primer desarrollo de la política medioambiental europea y establecen la senda que deben seguir los actos legislativos para estructurar la normativa europea en la materia.

El presente texto analiza la evolución de los fundamentos y objetivos de la normativa medioambiental europea, con especial atención a los PMA. Como se 
comprobará, de una situación inicial de inexistencia de política medioambiental en los Tratados constitutivos, se ha pasado por diferentes etapas en las que los primeros PMA y el AUE establecieron las bases de la misma. A continuación, el análisis se detendrá en los Tratados de Maastricht y Ámsterdam, que fortalecieron e impulsaron dicha política, algo que quedó reflejado de manera evidente en los PMA quinto y sexto. Seguidamente, se presentarán los objetivos, principios y directrices del Derecho ambiental europeo vigentes en la actualidad, determinados por el Tratado de Lisboa y el séptimo PMA. Finalmente, se estudiarán las líneas a seguir por parte de la UE en el futuro inmediato, recogidas en el Pacto Verde Europeo y en el octavo PMA, para cuya regulación se están desarrollando las negociaciones interinstitucionales correspondientes mientras se escriben estas líneas.

\section{ANTECEDENTES}

Los Tratados fundacionales de las CCEE no regularon el establecimiento de una política común de medio ambiente. No existía ninguna atribución expresa a las Comunidades para que actuasen en materia ambiental, ni figuraba entre los objetivos de las mismas la protección del medio ambiente 1 . Sin embargo, lo cierto es que los entonces seis Estados miembros venían aplicando normas nacionales con contenido medioambiental que podían crear obstáculos a los intercambios y a la libre circulación de mercancías que se había puesto en marcha en 1968, con la instauración de una zona de libre comercio. Ante esta situación, "las instituciones comunitarias intentaron conciliar las necesidades de la política industrial y la tutela del medio ambiente" recurriendo a la posibilidad que

\footnotetext{
${ }^{1}$ Los Tratados no recogían "ninguna manifestación normativa expresa que tratase directamente del medio ambiente o la política ambiental". DE CARVALHO LEAL, V., "El Medio Ambiente como objeto de protección jurídica en el ámbito comunitario: hacia un sistema de Responsabilidad Ambiental", Nuevo Derecho, vol. 4, núm. 4, 2009, p. 13. No obstante, era posible encontrar algún precepto que indirectamente conllevaba la protección del medio ambiente, como el artículo 35 del Tratado de la CEEA, que disponía que "cada Estado miembro creará las instalaciones necesarias a fin de controlar de modo permanente el índice de radioactividad de la atmósfera, de las aguas y del suelo". AlONSO GARCíA, E., El Derecho Ambiental de la Comunidad Europea, Madrid, Fundación Universidad-Empresa, 1993, p. 28.
} 
recogían los Tratados de armonización de las políticas de los Estados miembros que incidan en el funcionamiento del mercado común².

En esos primeros años, la Comunidad Económica Europea (CEE) sólo pudo "abordar la materia ambiental indirectamente, mediante disposiciones con otra finalidad directa, o, al menos, no refiriéndose directamente al medio ambiente"3. Así, puede decirse que tuvo lugar una expansión de competencias comunitarias por medio de una interpretación conjunta del Preámbulo del Tratado de la CEE, que asignaba como fin esencial de los Estados miembros "la mejora constante de las condiciones de vida y de trabajo de sus pueblos", y el artículo 2 que definía como objetivo la promoción de "un desarrollo armonioso de las actividades económicas en el conjunto de la Comunidad" y "una expansión continua y equilibrada", combinados con los artículos 100 y 235 que permitían al Consejo adoptar las disposiciones pertinentes para lograr el desarrollo del mercado común 4 . Es más, los fines citados "se consideraron objetivos de imposible logro sin el desenvolvimiento de una política de protección del medio ambiente"5.

Ahora bien, el objetivo inicial no fue tanto generar una normativa específica de protección ambiental, sino más bien aproximar "las legislaciones nacionales de los Estados miembros con el propósito de que las normas medioambientales de estos no incidieran en la libre competencia y en especial en el intercambio de

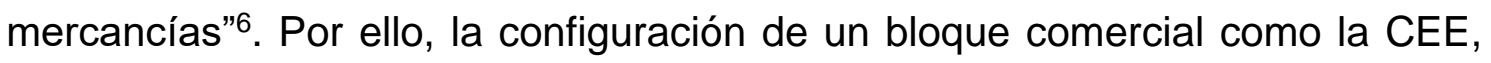
"formado por países con distintos niveles de calidad medioambiental, llevó a plantearse a los Estados miembros la conveniencia de adoptar una perspectiva supranacional en el diseño de las grandes líneas a seguir"7.

\footnotetext{
2 MOdAFFERI, C., "La política medioambiental europea. Estudio sobre el desarrollo de la protección ambiental y papel decisivo de la Unión Europea", Cuadernos Cantabria Europa, núm. 15, 2016, p. 56.

${ }^{3}$ FERNÁNDEZ DE GATTA SÁNCHEZ, D., "La política ambiental comunitaria: especial referencia a los programas de acción”, Revista de Instituciones Europeas, vol. 12, núm. 3, 1985, p. 727.

${ }^{4}$ Plaza Martín, C., Derecho Ambiental de la Unión Europea, Valencia, Tirant lo Blanch, 2005, p. 41.

${ }^{5}$ LÓPEZ Ramón, F., "Caracteres del Derecho Comunitario Europeo Ambiental", Revista de Administración Pública, núm. 142, 1997, p. 58.

${ }^{6}$ HINOJO ROJAS, M., "La política en materia de medio ambiente de la UE en perspectiva histórica: de Roma a Niza", Revista de Estudios Europeos, núm. 72, 2018, p. 27.

${ }^{7}$ MuÑoz De Bustillo, R. y BONETE, R., Introducción a la Unión Europea: un análisis desde la economía, Madrid, Alianza, 2002, p. 295.
} 
De este modo, la Comisión adoptó en 1971 el primer documento sobre esta materia ante la necesidad de tener presente el medio ambiente al objeto de regular adecuadamente el desarrollo del mercado común8. La Comisión abogaba por poner en marcha un programa general de acciones para reducir la contaminación, proteger el medio natural y armonizar las medidas ambientales. Siguiendo esta línea, los Jefes de Estado y de Gobierno de los países de las CCEE emitían en la Cumbre de París de octubre de 1972 una declaración política en la que se reconoció la necesidad de un crecimiento económico ligado a la mejora de la calidad de vida y a la protección del medio ambiente y de sus recursos ${ }^{9}$. Las CCEE seguían así la agenda internacional del momento, en la que había quedado reflejado el interés por el medio ambiente, tal y como se comprobó en la Conferencia de Naciones Unidas sobre el Medio Humano, celebrada pocos meses antes en Estocolmo. Entre los acuerdos adoptados destaca la conocida como Declaración de Estocolmo ${ }^{10}$, en la que los países reconocían por primera vez de modo general la necesidad de preservar los recursos naturales en beneficio de las generaciones presentes y futuras ${ }^{11}$.

Para cumplir con el mandato de la Cumbre de París, las CCEE desarrollaron una actuación fundamentada en una serie de principios recogidos en la primera comunicación de la Comisión sobre esta materia y que han guiado desde entonces la actividad comunitaria en el ámbito medioambiental ${ }^{12}$. En primer lugar, el compromiso de los Estados miembros de mejorar las condiciones de vida y de trabajo de sus ciudadanos, por medio de la promoción de un alto nivel

\footnotetext{
${ }^{8}$ Comisión Europea, comunicación de 22 de julio de 1971, Première communication sur la politique de la Communauté en matière de l'environnement, SEC (71) 2616 final.

${ }^{9}$ Texto íntegro del comunicado final de la Cumbre de París de los días 19 y 20 de octubre de 1972. Revista de Instituciones Europeas (1974), vol. 1, núm. 1, pp. 491-498.

${ }^{10}$ Naciones Unidas, "Declaración de la Conferencia de Naciones Unidas sobre el Medio Humano", contenida en el Informe de la Conferencia de Naciones Unidas sobre el Medio Humano, Nueva York (A/CONF.48/14/Rev.1), 1973. Se trata de un texto que "no es vinculante, pero ha tenido el mérito de haber comprometido éticamente a los países que la firmaron" y a partir del mismo "también el derecho interno de los Estados comienza a ser más cuidadoso a las problemáticas ambientales". MODAFFERI, C., "La política medioambiental..." cit., p. 53.
}

11 Como se ve, cabe afirmar que la europeización del medio ambiente, es decir, su institucionalización a nivel de las CCEE, comenzó justo después de la conferencia de Estocolmo. MEYER, J-H. y PONCHARAL, B., "L'européanisation de la politique environnementale dans les années 1970", Vingtième Siècle. Revue d'histoire, núm. 113, 2012, p. 121.

12 Girón LarruceA, J. A., La Comunidad Europea como Organización Internacional, Madrid, Centro de Estudios Ramón Areces, 1999, p. 114. 
de protección y de mejora de la calidad del medio ambiente. En segundo lugar, el buen funcionamiento del mercado interior, por lo que es preciso suprimir las diferencias en las legislaciones medioambientales de los Estados miembros que podrían ocasionar obstáculos a la libre circulación de mercancías. En tercer lugar, recurrir a la Comunidad como instrumento adecuado para adoptar o coordinar medidas para solucionar problemas medioambientales, caracterizados por su naturaleza internacional que supera los límites fronterizos de los países. En el mismo punto octavo de la declaración de París de 1972 en el que se fijaban los principios citados, se encargaba a las instituciones la elaboración de un programa de acción sobre medio ambiente.

\section{LOS PRIMEROS PROGRAMAS DE ACCIÓN SOBRE MEDIO AMBIENTE}

El Consejo aprobó el primer PMA en noviembre de $1973^{13}$, cuyo objetivo general era la mejora de la calidad de vida y del medio ambiente de los Estados miembros. Es de destacar que, sobre el papel, resultaba un primer paso esperanzador, no en vano se recogía expresamente que los objetivos del artículo 2 del Tratado CEE -la promoción de un desarrollo armonioso de las actividades económicas y una expansión continua y equilibrada- no podrían considerarse en adelante sin una lucha eficaz contra la contaminación y la protección del medio ambiente.

Aunque este primer PMA "contenía ciertas medidas preventivas, su principal función era contrarrestar los efectos de la contaminación y el deterioro ambientales" ${ }^{14}$, tal y como se aprecia en los objetivos establecidos. Eran los siguientes: reducción de la contaminación atmosférica y de los vertidos a las aguas; utilización racional de los recursos naturales; mantenimiento de un equilibrio ecológico; orientación del desarrollo en función de las exigencias de calidad medioambiental; propuesta en el marco de las Organizaciones internacionales de soluciones comunes a los problemas del medio ambiente.

\footnotetext{
${ }^{13}$ Declaración del Consejo de las CCEE y de los representantes de los gobiernos de los Estados miembros reunidos en el seno del Consejo, de 22 de noviembre de 1973, relativa a un Programa de acción de las Comunidades Europeas en materia de medio ambiente, DOCE C 112, de 20 de diciembre de 1973.

${ }^{14}$ FernándeZ De GatTA SÁNCHEZ, D., "La política ambiental comunitaria...", cit., p. 740.
} 
Para el logro de estas metas, el Programa establecía una serie de principios que debían vertebrar la política medioambiental: prevención y toma de medidas para evitar los daños; evaluación del impacto ambiental; mejora de los conocimientos científicos y técnicos; principio de "quien contamina paga"; fomento de una opinión pública de protección medioambiental.

La mayor virtud de este primer PMA puede resumirse en que evidenció la interdependencia existente entre el desarrollo y la prosperidad económicos y la protección del medio ambiente ${ }^{15}$. Ahora bien, este primer programa tuvo que enfrentarse a un contexto adverso marcado por la crisis del petróleo que estalló en octubre de 1973 y la consiguiente recesión económica. Además, debe subrayarse que adolecía de la falta de concreción de medios o instrumentos operativos para cumplir las metas previstas. Lo cierto es que las definiciones principales contenidas en el programa, la manera de describir los problemas y las soluciones previstas reflejan que las CCEE aún no disponían de un criterio propio para actuar en esta materia y se inspiraron en el consenso internacional emergente iniciado con la conferencia de Naciones Unidas de 1972 a la que nos hemos referido en el epígrafe anterior ${ }^{16}$.

En mayo de 1976 el Consejo aprobó el segundo PMA ${ }^{17}$, el cual es considerado como una continuación del anterior, puesto que ambos "pusieron los pilares para la defensa de los intereses ambientales en la Comunidad y fijaron como gran objetivo la utilización racional de los recursos naturales compatible con el desarrollo económico" ${ }^{18}$. No obstante, aunque el nuevo programa dedicaba mayor atención a la lucha contra la contaminación, "incluyó ya algunas

\footnotetext{
15 PopeanGA, V., "Environmental action programs in the European Unión - Evolution and Specific", Annals of the "Constantin Bránçusi" University of Tárgu Jiu, Letter and Social Science Series, núm. 3, 2013, p. 25.

${ }^{16}$ MeYer, J-H. y PoncharaL, B., "L'européanisation...", cit., p. 123.

${ }^{17}$ Resolución del Consejo de las CCEE y de los representantes de los gobiernos de los Estados miembros reunidos en el seno del Consejo, de 17 de mayo de 1976, relativa a la prosecución de una política y de un programa de acción de las Comunidades Europeas en materia de medio ambiente, DOCE C 139, de 13 de junio de 1977.

${ }^{18}$ Cuenca García, E., Economía de la Unión Europea, Madrid, Pearson-Prentice Hall, 2007, p. 419.
} 
referencias preventivas (en particular, la previsión de las evaluaciones de impacto ambiental) y a más largo plazo"19.

Los objetivos particulares de este programa consistían en la puesta en marcha de un procedimiento de información en materia medioambiental y la aplicación efectiva del principio "quien contamina paga", todo ello, remarcando la necesidad de tomar medidas de carácter preventivo, mediante el establecimiento de procedimientos adecuados para realizar estudios de impacto medioambiental. De este modo, el Segundo PMA incidió en la necesidad de establecer normas sobre calidad del medio ambiente, sobre calidad de los productos, sobre procedimiento para la construcción de instalaciones fijas y sobre explotación. Se trataba, como se ve, de impedir desde el origen la contaminación más que buscar soluciones una vez producido el daño ambiental.

Como se ha mencionado arriba, los Tratados constitutivos no contenían una regulación medioambiental común para los Estados miembros, lo que provocó que la existencia de diferentes legislaciones nacionales alterara las reglas de la libre competencia. Ante esta situación, la aplicación y puesta en marcha de los dos primeros PMAs supuso la aprobación de numerosas directivas para aproximar las legislaciones estatales en materias tales como la contaminación hídrica y atmosférica, el ruido y los residuos, fijando así unos estándares comunitarios iniciales de calidad ambiental. Sin ellos, "sería imposible que posteriormente hubieran podido plantearse alternativas de actuación comunitarias basadas en los mecanismos del mercado"20.

A lo largo de la década de 1980 la protección del medio ambiente fue adquiriendo mayor protagonismo en el seno de las CCEE. En ello va a tener notable influencia el rol de líder que en este ámbito adquirió Alemania, impulsando la adopción de nuevas medidas desde la propia CEE, así como que el conjunto de los Estados miembros avanzase en la misma dirección ${ }^{21}$. No obstante, debe destacarse que al inicio de la década, en un contexto de grave crisis económica,

\footnotetext{
${ }^{19}$ Fernández De GatTa SánCheZ, D., "El Séptimo Programa Ambiental de la Unión Europea, 2013-2020", Revista Aragonesa de Administración Pública, núm. 41-42, 2013, p. 73.

${ }^{20}$ LÓPEZ RAMÓN, F., “Caracteres del Derecho...”, cit., p. 56.

${ }^{21}$ Alberton, M., "Environmental Protection in the EU Member States: Changing Institutional Scenarios and Trends", L'Europe en formation, núm. 363, 2012, p. 290.
} 
surgió la cuestión de si la política ambiental europea "debía modificarse o no, para acomodarla, restrictivamente, a la crisis" y lo cierto es que "se modificó, efectivamente, pero en vez de reducirla se potencia" mediante el Tercer PMA ${ }^{22}$. El nuevo programa fue aprobado por el Consejo en de febrero de $1983^{23}$, siendo su objetivo general continuar la línea iniciada por los dos primeros Programas, especialmente reforzando el carácter preventivo que aportó el segundo de ellos, si bien introdujo importantes cambios cualitativos que marcaron el contenido de los posteriores planes de acción.

Junto a las directivas que continuaron el proceso de fijación de los estándares de calidad ambiental en relación con los diversos recursos naturales, se aumentó el control de impacto medioambiental de las políticas comunitarias, fortaleciendo así el principio de prevención, al generalizar la exigencia del examen de las consecuencias sobre el medio ambiente de proyectos significativos, especialmente en agricultura e infraestructuras. Al mismo tiempo, se fijó el perfil exacto del principio "quien contamina paga", introduciendo la obligación de restauración. Finalmente, cabe mencionar también la materialización de un mayor énfasis en la sensibilización en torno a la necesaria protección del medio ambiente y la creación de ayudas a los esfuerzos de I+D en materia medioambiental.

Las autoridades comunitarias fueron conscientes de la creciente preocupación entre la opinión pública de la necesidad de proteger y gestionar adecuadamente los recursos naturales. Por ello, se establecieron acciones encaminadas a conservar y proteger mejor los ecosistemas naturales y asegurar una gestión integrada de las regiones especialmente sensibles debido a su importancia ambiental o a su atractivo para el desarrollo económico.

Una de las grandes aportaciones del tercer PMA vino determinada por el hecho de que a partir del mismo se identificó un objetivo general de protección del medio ambiente para el conjunto de las políticas comunitarias. Durante el

\footnotetext{
22 FernándeZ De GATTA SÁNCHEZ, D., "La política ambiental comunitaria...", cit., p. 745.

${ }^{23}$ Resolución del Consejo de las CCEE y de los representantes de los gobiernos de los Estados miembros, reunidos en el seno del Consejo, de 7 de febrero de 1983, relativa a la prosecución y ejecución de una política y de un programa de acción de las CCEE en materia de medio ambiente (1982-1986), DOCE C 46, de 17 de febrero de 1983.
} 
desarrollo de la aplicación del programa se constató que las consideraciones ambientales incidían sobre determinadas políticas como agricultura, energía, industria, transportes o turismo, por lo que se veía necesario la integración de las preocupaciones del medio ambiente en las demás políticas comunitarias. Se producía un evidente avance con respecto a la situación inicial, puesto que el problema central en esta materia "dejó de ser la existencia de divergencias normativas entre los Estados miembros que pudieran afectar al buen funcionamiento del mercado común. La preocupación por la calidad del medio ambiente se insertaba así en el mismo concepto de la economía"24.

Estas consideraciones estuvieron presentes en las negociaciones que dieron lugar al Acta Única Europea y fundamentan el avance que este texto traería en la materia que nos ocupa.

\section{EL ACTA ÚNICA EUROPEA Y EL CUARTO PMA}

El 1 de julio de 1987 entró en vigor el Acta Única Europea (AUE), texto firmado un año antes por los entonces doce Estados miembros y por medio del cual se reformaban los Tratados constitutivos de las CCEE con el objetivo de relanzar la integración europea. Además de aportar relevantes novedades institucionales y de establecer las medidas necesarias para la puesta en marcha del mercado interior, el AUE introdujo en el ámbito europeo la regulación de materias estructuradas como nuevas políticas, tal y como sucedió con el medio ambiente. De este modo, "consagró como derecho originario (europeo) las bases para la protección ambiental" 25 .

Con este texto, los líderes de las CCEE comenzaban a buscar una respuesta ante los retos que planteaba la propia ciudadanía, cuya presión a favor de la protección medioambiental crecía ante los desastres ecológicos cada vez mayores y en especial ante la catástrofe de Chernóbil ${ }^{26}$. En cierta manera, los

\footnotetext{
${ }^{24}$ Cuenca García, E., Economía de la Unión..., cit., p. 421.

${ }^{25}$ De Carvalho Leal, V., "El Medio Ambiente...", cit., p. 14.

${ }^{26}$ WOOD, D. M. y YESILADA, B. A., The Emerging European Union, New York, Pearson Longman, 2006, p. 172.
} 
actores sociales demandaban la europeización de las políticas ambientales ${ }^{27}$. Como veremos a continuación, el AUE dio cierta respuesta a dicha demanda y constituyó un hito en la evolución del papel de la Comunidad en la protección medioambiental, al introducir una normativa específica y el principio de subsidiariedad en esta materia, relacionándola además con otras como el comercio, la agricultura, la energía o la investigación ${ }^{28}$.

El Acta Única incorporó en la Tercera Parte del Tratado de la CEE una normativa específica sobre Medio Ambiente (entonces, el Título VII) ${ }^{29}$, paliando así "la mayor dificultad que se ha planteado a la Comunidad Europea" que en esta materia "ha sido la imprecisión de bases jurídicas en los tratados comunitarios y sobre todo en el Tratado CEE"30. Sobre la base de la atribución de competencias consolidó de manera definitiva la base jurídica de la Comunidad en materia ambiental, estableciendo el esquema normativo que se mantiene en la actualidad $^{31}$. De esta manera, el AUE "instaló la conservación del medio ambiente en el derecho primario de la CE y aludió a su condición inherente al desarrollo económico y social, la calidad de vida y la sostenibilidad de otras políticas europeas" 32 . Como se ve, "se trataba de preparar y poner en práctica programas para conseguir una dimensión ecológica del desarrollo económico"33.

De los tres artículos que compusieron el nuevo Título (entonces $130 \mathrm{R}, 130 \mathrm{~S}$ y $130 \mathrm{~T}$ ), el primero recogió las ideas fundamentales que se habían trabajado en los programas de acción. Así, se fijaron como objetivos de la Comunidad en materia medioambiental: la conservación, protección y mejora de la calidad del medio ambiente; la protección de la salud de las personas; y la utilización

\footnotetext{
${ }^{27}$ MeYer, J-H. y PoncharaL, B., "L'européanisation..." cit., p. 125.

${ }^{28}$ GUSEV, A. S., "Climate Change Issues in a Transatlantic Context", L'Europe en formation, núm. 360,2011 , p. 80.

${ }^{29}$ La implementación en el Tratado de un capítulo propio para la regulación de la política europea de medio ambiente es considerada un punto de inflexión para la misma, al adquirir un nuevo status. POPEANGA, V., "Environmental..." cit., p. 26.

30 TorRes UgenA, N., "La protección del medio ambiente en el Acta única Europea", Noticias de la CEE, núm. 54, 1989, p. 94.

${ }^{31}$ FeRnández De GatTA SÁNCHEZ, D., "Política ambiental de la Unión Europea", en LóPEZ Ramón, F. (coord.), Observatorio de Políticas Ambientales 2006, Madrid, CIEMAT, 2006, p. 95.

${ }^{32}$ Nieto Solís, J. A., La Unión Europea. Una nueva etapa en la integración económica de Europa, Madrid, Pirámide, 2005, p. 217.

${ }^{33}$ Cuenca García, E., Economía de la Unión..., cit., p. 412.
} 
prudente y racional de los recursos naturales. Para el logro de los mismos, la acción de la Comunidad debía basarse en los principios de acción preventiva, de corrección de los ataques al medio ambiente (preferentemente en la fuente misma) y de quien contamina paga. Asimismo, el precepto especificó que "las exigencias de la protección del medio ambiente serán un componente de las demás políticas de la Comunidad", tal y como se había determinado con el tercer PMA.

La nueva normativa fundamental europea determinó además que en la elaboración de la acción medioambiental europea se tendrían en cuenta: los datos científicos y técnicos disponibles; las condiciones del medio ambiente en las diversas regiones de la Comunidad; las ventajas y las cargas que puedan resultar de la acción o de la falta de acción; el desarrollo económico y social de la Comunidad en su conjunto y el desarrollo equilibrado de sus regiones.

Finalmente, debe destacarse que el AUE estableció el principio de subsidiariedad como referente de actuación en este ámbito, al delimitar que la acción comunitaria en materia de medio ambiente tendría lugar en la medida en que los objetivos puedan conseguirse en mejores condiciones en el plano comunitario que en el de los Estados miembros considerados aisladamente.

Poco después de la entrada en vigor del AUE y con una evidente influencia del mismo, el Consejo aprobó en octubre de 1987 el cuarto PMA ${ }^{34}$. Este programa venía claramente influenciado e impulsado por dos elementos. Por un lado, la decisión del Consejo Europeo de pasar a considerar la protección del medio ambiente como una herramienta que podría suponer crecimiento económico y creación de empleo, frente a hipótesis vigentes hasta entonces en sentido contrario ${ }^{35}$. Por otro lado, el propio AUE que, como se ha dicho, preveía explícitamente el desarrollo de una política común de medio ambiente y que las exigencias medioambientales debían pasar a ser parte de las demás políticas comunitarias.

\footnotetext{
${ }^{34}$ Resolución del Consejo de las CCEE y de los representantes de los gobiernos de los Estados miembros, reunidos en el seno del Consejo, de 19 de octubre de 1987, relativa a la continuación y aplicación de una política y de un programa de acción de las CCEE en materia de medio ambiente (1987-1992), DOCE C 289, de 29 de octubre de 1987.

${ }^{35}$ Consejo Europeo de Bruselas de 29 y 30 de marzo de 1985 - Conclusions, SN 1381/2/85.
} 
De este modo, aunque el nuevo programa se inscribía dentro de la estrategia global de su precedente, presentaba un carácter más completo y expansivo, derivado de la nueva normativa fundamental instaurada por el AUE. Así, continuó con la línea iniciada durante la aplicación del tercer PMA, a saber, la integración en otras políticas comunitarias de los aspectos medioambientales y se preocupó por el grado de cumplimiento de los dos primeros programas. A este respecto, se propusieron medidas para asegurar el alcance de las metas en los diferentes Estados miembros como el fomento de la educación ambiental, el establecimiento de normas de calidad ambiental más estrictas y el acceso general a la información sobre temas ambientales. Es de destacar el hecho de que la Comisión se comprometió a elaborar procedimientos que garantizasen que las necesidades de protección medioambiental fuesen tenidas en cuenta en las actividades financiadas por los fondos estructurales.

Precisamente, el papel de la Comisión, encargada de poner en marcha la política medioambiental en cuanto responsable de las propuestas legislativas que el Consejo convertiría en actos jurídicos, cobraba especial relevancia. Más aún en un momento en que se dudaba de la efectividad del principio de quien contamina paga ${ }^{36}$ y de la actitud de los Estados miembros a la hora de facilitar la información para que la Comisión controlase el cumplimiento de las normas medioambientales ${ }^{37}$. Por ello, el cuarto PMA facilitó la utilización de instrumentos económicos para la gestión ambiental como un sistema de responsabilidad objetiva ampliada para los daños causados al medio ambiente.

Finalmente, el programa inició la creación de los instrumentos necesarios para el desarrollo estable de la política ambiental. Así, entre los elementos organizativos debe destacarse la creación de la Agencia Europea de Medio

\footnotetext{
${ }^{36}$ Este principio "ha sido objeto de controversia a lo largo del tiempo pudiéndose afirmar a la vista de la producción normativa comunitaria que ha cobrado fuerza como eje de la política medioambiental aunque su aplicación efectiva sigue siendo una cuestión por hacer". ARMENGOL y Butrón De MúGICA, D. y CARRILlo VARGAS, M. C., "El Cuarto Programa de la CEE en materia de medio ambiente (1987/1992) y la política ambiental comunitaria", Estudios Regionales, núm. 26, 1990, p. 204.

${ }^{37}$ Precisamente, uno de los problemas que el programa se encontraría a la hora de cumplir sus objetivos sería "la falta de solidaridad de los Estados miembros al anteponer los intereses nacionales frente a los comunitarios". Ibídem, p. 207.
} 
Ambiente (AEMA) en 199038, que se pondría en marcha cuatro años más tarde, y el instrumento financiero específico para la protección del medio ambiente LIFE en $1992^{39}$. También en 1992 se creó la red Natura $2000^{40}$, que se ha convertido en uno de los principales instrumentos para la conservación de la naturaleza en la UE. Su objetivo consiste en asegurar la supervivencia a largo plazo de las especies y los hábitats más amenazados de Europa y detener así la pérdida de biodiversidad. Consta de un conjunto de áreas protegidas en las que se posibilita la coexistencia de actividades humanas sostenibles con la flora, fauna y territorios en situación de vulnerabilidad.

\section{EL TRATADO DE MAASTRICHT Y EL QUINTO PMA}

El Consejo Europeo determinó en junio de 1990 el camino que seguiría la reforma de los Tratados en esta materia, al establecer que la acción de la Comunidad y sus Estados miembros se desarrollase sobre los principios del desarrollo sostenible y de una actuación preventiva con respecto del medio ambiente $^{41}$. El Consejo Europeo reconocía que, ante el fuerte aumento del crecimiento económico que se esperaba a partir de 1992 por la realización del mercado interior, debían acelerarse los esfuerzos para garantizar que dicho crecimiento fuese sostenible y no perjudicase al medio ambiente. Así, ante los riesgos derivados de una mayor producción y una demanda creciente de transporte, energía e infraestructura, abogaba por integrar plena y eficazmente las consideraciones medioambientales en las políticas reguladoras de esos y otros sectores. Resultaba evidente la influencia que estaban consiguiendo

\footnotetext{
${ }^{38}$ Reglamento (CEE) no 1210/90 del Consejo, de 7 de mayo de 1990, por el que se crea la Agencia Europea de Medio Ambiente y la Red Europea de Información y Observación sobre el Medio Ambiente.

${ }^{39}$ Se comenzaba así a paliar la falta de recursos financieros que podía tener la que entonces se consideraba "una política emergente que se desarrolla en períodos de prosperidad económica pero que también es la primera víctima cuando los recursos disminuyen". TORRES UGENA, N., "La protección del medio ambiente...", cit., p. 105.

${ }^{40}$ La red Natura 2000 tiene su origen en la Directiva 92/43/CEE del Consejo, de 21 de mayo de 1992, relativa a la conservación de los hábitats naturales y de la fauna y flora silvestres, DOCE L 206, de 22 de julio de 1992.

${ }^{41}$ Consejo Europeo de Dublín de 25 y 26 de junio de 1990 - El Imperativo del Medio Ambiente, Declaración contenida como Anexo Il de las Conclusiones de la Presidencia, SN 60/1/90.
} 
ejercer aquellos Estados miembros más avanzados en la tarea de hacer compatible el crecimiento económico y el respeto al medio ambiente ${ }^{42}$.

La segunda reforma de los Tratados constitutivos llegó el 7 de febrero de 1992, con la firma del Tratado de Maastricht, que entró en vigor el 1 de noviembre de 1993. El nuevo Tratado recogía la constitución de la UE sobre el fundamento de las CCEE, estableciendo como nuevo texto jurídico al TUE, que se unía a los tres Tratados que regulaban las Comunidades, los cuales recibían importantes innovaciones y modificaciones. En cuanto a la materia que nos ocupa, el TUE estableció entre los objetivos de la ahora ya UE "promover un progreso económico social equilibrado y sostenible" (art. 2 TUE). Este objetivo se concretó en la reforma que se realizó en el artículo 2 del Tratado de la Comunidad Europea (TCE), donde se recogían los fines a lograr mediante el establecimiento de un mercado común y una unión económica y monetaria. Junto al desarrollo económico equilibrado, la convergencia económica, un alto nivel de empleo y de protección social y la elevación de la calidad de vida, se citaba explícitamente un crecimiento sostenible que respete el medio ambiente.

La regulación del medio ambiente recogida en el TCE -que pasó a ser Título XVI- recibió varias modificaciones. En primer lugar, a los tres objetivos especificados por el AUE se sumaron el fomento de medidas a escala internacional destinadas a hacer frente a los problemas regionales o mundiales del medio ambiente, así como alcanzar un elevado nivel de protección en esta materia. En segundo lugar, a los principios en los que debe basarse la acción de la UE se añadió el de cautela. En tercer lugar, el procedimiento de consulta fue sustituido, para la mayoría de decisiones a adoptar en materia medioambiental, por el procedimiento de cooperación, lo que posibilitaba cierta participación del Parlamento Europeo (PE) a la hora de legislar. En cambio, los PMA pasaban a ser adoptados por medio del nuevo procedimiento de codecisión, en el que el PE y el Consejo legislan conjuntamente.

\footnotetext{
${ }^{42}$ El caso más evidente era el de Dinamarca. Su preocupación por la protección del medio ambiente era similar a la existente en los otros países nórdicos que a comienzos de los años 90 se encontraban negociando su adhesión a la CE. Precisamente, la presencia como observadores de Suecia, Finlandia y Noruega (país que no llegó a ingresar) en las negociaciones que darían lugar al Tratado de Maastricht facilitó que la cuestión medioambiental estuviese presente en la correspondiente Conferencia Intergubernamental.
} 
Finalmente, el Tratado de Maastricht abrió la posibilidad de dotar recursos comunitarios para la defensa del medio ambiente. En este ámbito, se tuvo presente una consideración que se hacía evidente desde tiempo atrás, referida al hecho de que estando la UE formada por países con distinto grado de desarrollo económico, "cuando se plantea la necesidad de garantizar unos mínimos medioambientales, se hace imprescindible la puesta en marcha de alguna compensación que favorezca a aquellos países con un grado de desarrollo más bajo, que sin embargo, y por ello mismo, estarían adoptando una normativa medioambiental demasiado avanzada para su nivel de desarrollo"43. Ante esta situación, se plantea la creación de fondos de ayuda económica para que dichos países puedan alcanzar niveles de respeto medioambiental propios de países más desarrollados ${ }^{44}$.

Tras el Tratado de Maastricht llegó el quinto PMA ${ }^{45}$. Su objetivo general era orientar progresivamente el modelo de crecimiento europeo hacia el desarrollo sostenible, mediante el cambio de las pautas de producción y consumo. Se trataba de crear el marco adecuado para un nuevo planteamiento del medio ambiente y de la actividad y desarrollo económico y social. De este modo, se pretendía la instauración en la UE de una política medioambiental horizontal, es decir, "una política capaz de extender su contenido hacia todas las acciones de la Unión y de sus Estados miembros, con el fin de evitar, en la medida de lo posible, consecuencias graves sobre los ecosistemas y la calidad de vida de los ciudadanos"46.

Este programa, partiendo de la experiencia de los cuatro primeros, establecía las bases para una nueva perspectiva de protección medioambiental generalizada a todos los ámbitos en los que la UE tenía competencias, es decir,

\footnotetext{
${ }^{43}$ Muñoz De Bustillo, R. y Bonete, R., Introducción a la Unión..., cit., p. 297.

${ }^{44}$ De este modo, junto al instrumento LIFE mencionado arriba, se comenzó a destinar una parte importante de la capacidad financiera de los Fondos Estructurales y del Fondo de Cohesión a la protección del medio ambiente.

${ }^{45}$ Resolución 93/C 138/01, de 1 de febrero de 1993, del Consejo y de los representantes de los gobiernos de los Estados miembros reunidos en el seno del Consejo, sobre un Programa comunitario de política y actuación en materia de medio ambiente y desarrollo sostenible, DOCE C 138, de 17 de mayo de 1993. Aunque el Tratado de Maastricht introdujo el procedimiento de codecisión para adoptar los PMA, al no haber entrado en vigor aún quedó en manos del Consejo la adopción del quinto PMA.

${ }^{46}$ Nieto Solís, J. A., La Unión Europea..., cit., p. 218.
} 
pretendía la integración plena de la política de medio ambiente en las demás políticas europeas, si bien concentró su acción en cinco sectores prioritarios, a saber, industria, energía, transportes, agricultura y turismo. Destacamos los siguientes objetivos concretos: el desarrollo de una gestión racional de los recursos en las empresas; la mejora de la eficacia energética; la reducción del consumo de combustibles fósiles; la promoción de las energías renovables; el desarrollo de los transportes públicos; la vigilancia del deterioro como consecuencia del aumento de los cultivos intensivos, la utilización de abonos y la acumulación de excedentes; por último, la mejora de la gestión del turismo de masas y la promoción de formas alternativas de turismo.

Para la consecución de tales objetivos, el quinto PMA estructuró numerosos instrumentos, entre los que cabe citar: la creación de mecanismos para la elaboración de indicadores medioambientales; el incremento de las inversiones en actividades de I+D relativas a medio ambiente y energía; la integración de los requisitos de protección medioambiental en las políticas fiscales; el aumento de la financiación en el seno de los Fondos Estructurales y de Cohesión, así como en el Programa LIFE; un incremento sustancial de la información pública sobre medio ambiente facilitando el acceso a la misma; y medidas en materia de educación (básica, universitaria y profesional).

Durante este período, concretamente en 1994, comenzó a ser operativa la AEMA. Se trata del organismo comunitario dedicado a proporcionar información sólida e independiente acerca del medio ambiente, en el que pueden participar, además de los Estados miembros de la UE, otros países europeos ${ }^{47}$. Siguiendo las pautas recogidas en el Tratado, la AEMA trata de facilitar a la UE y a los países miembros la toma de decisiones destinadas a mejorar el medio ambiente, integrando las consideraciones medioambientales en las políticas económicas y avanzando en materia de sostenibilidad ${ }^{48}$.

\footnotetext{
${ }^{47}$ En la actualidad, junto a los 27 Estados miembros de la UE, son miembros de la AEMA Islandia, Liechtenstein, Noruega, Suiza y Turquía. Son países colaboradores Albania, BosniaHerzegovina, Kosovo, Macedonia del Norte, Montenegro y Serbia.

${ }^{48}$ Para desarrollar sus funciones la AEMA elabora programas plurianuales en los que determina la estrategia a seguir en su actuación y realiza periódicamente informes de evaluación sobre el estado del medio ambiente en la UE. Todo los informes y documentos de la AEMA son accesibles en su página web: https://www.eea.europa.eu/publications
} 
Precisamente, en 1995 la AEMA hizo público el informe de revisión del quinto PMA, en el que hacía un repaso de los avances logrados hasta entonces por la UE, destacando que se había conseguido reducir algunas de las presiones a que se somete al medio ambiente. Sin embargo, la Agencia consideraba entonces que con dichas medidas "no se conseguirá la integración plena de las consideraciones medioambientales en los sectores económicos, ni tampoco el desarrollo sostenible"49. Así, entre los positivos logros obtenidos por los cinco primeros PMA debe destacarse la adopción de normas comunitarias para reducir la contaminación, la puesta en práctica de instrumentos de intervención en materia ambiental, la realización y coordinación de investigaciones, la sensibilización de la opinión pública, y el mantenimiento de una postura única de la UE en esta materia en el contexto internacional. En cuanto a las lagunas, hay que subrayar la desproporción notable entre los programas y los medios disponibles, lo que supuso que en ocasiones los resultados no alcanzasen el objetivo inicial; las diferentes técnicas y métodos existentes entre los Estados miembros, algunos muy avanzados con respecto a los demás; y la existencia de diferentes puntos de vista en materia de medio ambiente, por lo que en ocasiones la postura única de la UE lo ha sido de mínimos.

\section{EL TRATADO DE ÁMSTERDAM Y EL SEXTO PMA}

El 2 de octubre de 1997 se firmó el Tratado de Ámsterdam que entró en vigor el 1 de mayo de 1999. Aunque recibió numerosas críticas, es necesario reconocer que en el ámbito del medio ambiente aportó relevantes novedades, articulando "de una forma orgánica y sistematizada la regulación de esta política" ${ }^{50}$. En lo que al TUE se refiere, introdujo en su preámbulo el principio de desarrollo sostenible, de modo que los Estados miembros, en su decisión de promover el progreso económico y social de sus pueblos, tendrán en cuenta dicho principio. De este modo, al subrayar el desarrollo sostenible como una de las principales

\footnotetext{
${ }^{49}$ Agencia Europea del Medio Ambiente, El medio ambiente en la UE - Informe para la revisión del quinto programa de acción sobre el medio ambiente, Luxemburgo, OPOCE, 1995, p. 9.

50 Girón LARRuCEA, J. A., La Comunidad Europea..., cit., p. 115.
} 
metas de la UE, quedaba establecido como objetivo del proceso de integración europea y no exclusivamente de la política de medio ambiente ${ }^{51}$.

En cuanto a las novedades introducidas en el TCE, en el artículo 2 se realizaron varias adiciones a la misión de la UE, la cual promoverá "un desarrollo armonioso, equilibrado $y$ sostenible de las actividades económicas", y "un alto nivel de protección y de mejora de la calidad del medio ambiente". Como se ve, se distinguen dos objetivos: por un lado, la sostenibilidad, entendida como "un modo de relacionarse con los recursos naturales y ambientales (un modo 'amigable', podríamos decir) que constituye una condición necesaria para un desarrollo económico perdurable a largo plazo"; por otro, "la mejora del medio ambiente como una condición necesaria para el avance en la calidad y nivel de vida de los ciudadanos"52.

A destacar la creación un nuevo precepto por el que se impulsaba la generalización de la protección medioambiental como fin para tener en cuenta en todas las políticas europeas. El entonces nuevo artículo 3C TCE (luego artículo 6) recogía de forma específica que "las exigencias de la protección del medio ambiente deberán integrarse en la definición y en la realización de las políticas y acciones de la Comunidad (...), en particular con objeto de fomentar un desarrollo sostenible" ${ }^{53}$. De este modo, se materializaba en el derecho primario europeo la acción horizontal y transversal que el quinto PMA había iniciado, al establecerse que las cuestiones medioambientales se consideran obligatorias para el desarrollo de otras políticas europeas ${ }^{54}$.

La Política de Medio Ambiente se mantuvo regulada en un Título propio del TCE, que pasaba a ser el Título XIX (tras la nueva numeración, artículos 174 a 176). El Tratado de Ámsterdam aportaba una importante novedad en cuanto al procedimiento legislativo a seguir para adoptar las normas medioambientales europeas, que pasaba a serlo de modo general la codecisión, en lugar del

\footnotetext{
${ }^{51}$ WoOd, D. M. y YeSILADA, B. A., The emerging..., cit., p. 172.

52 YÁBAR SterliNG, A., "El desarrollo sostenible, principio y objetivo común de la sociedad y el mercado, en la UE de nuestros días", Foro. Revista de ciencias jurídicas y sociales, núm. 0, 2004, p. 82 y 83.

${ }^{53}$ Esta especificación suponía la desaparición de la correspondiente mención que en el mismo sentido el TUE recogió en el artículo 130 R (174 con la nueva numeración).

54 Popeanga, V., "Environmental...", cit., p. 24.
} 
procedimiento de cooperación, posibilitando así un mayor grado de intervención del PE en materia de medio ambiente y, por tanto, un relevante avance en la democratización de esta política.

El Tratado de Niza no estableció modificación alguna en materia de medio ambiente. En cambio, sí reformó la estructura de la UE para posibilitar la adhesión de una docena de países en los siguientes años. Las autoridades europeas eran conscientes de que la ampliación condicionaría la política medioambiental de la UE, "al incorporarse países con un historial de respeto al medio ambiente inferior, en la mayoría de los casos, al practicado por casi todos los Estados miembros" ${ }^{55}$. Por ello, dentro de la obligación de incorporar plenamente el acervo comunitario que tiene todo Estado para poder ser miembro de la UE, se hizo especial hincapié en que los nuevos socios adaptasen a nivel interno toda la legislación medioambiental de la UE.

A la par que se desarrollaban las negociaciones que darían lugar al Tratado de Niza, la UE dio a conocer la Estrategia de Lisboa, un plan global que tenía por objetivo convertir Europa en la economía del conocimiento más competitiva y dinámica del mundo para 2010. Aunque inicialmente no se recogió, la dimensión ambiental fue incorporada en el Consejo Europeo de Gotemburgo de junio de 2001, estableciendo la Estrategia Europea para el Desarrollo Sostenible ${ }^{56}$. La estrategia reafirmaba el desarrollo sostenible como objetivo fundamental de los Tratados — por tanto, del proceso de integración europea-y exigía "utilizar las políticas económicas, sociales y ambientales de forma interactiva" (párrafo 19), de modo que todos los sectores económicos, especialmente energía y transportes, "participen en el desarrollo y una mayor utilización de nuevas tecnologías favorables al medio ambiente" (párrafo 21).

Siguiendo las pautas marcadas por el Consejo Europeo de Gotemburgo, en julio de 2002 se aprobó el sexto $\mathrm{PMA}^{57}$, siendo el primero aprobado por medio del procedimiento de codecisión. El programa determinaba cuatro ámbitos de

\footnotetext{
${ }^{55}$ MuÑoz De Bustillo, R. y BOnete, R., Introducción a la Unión..., cit., p. 303.

56 Consejo Europeo de Gotemburgo de 15 y 16 de junio de 2001 - Conclusiones de la Presidencia, SN 2001/1/01 REV 1.

57 Decisión no 1600/2002/CE del PE y del Consejo, de 22 de julio de 2002, por la que se establece el Sexto Programa de Acción Comunitario en Materia de Medio Ambiente, DOCE L 242, de 10 de septiembre de 2002.
} 
actuación, estableciéndose en cada uno el objetivo a lograr. El primero de ellos, considerado el principal reto, era el cambio climático, ámbito en el que se determinó como meta a corto plazo alcanzar los objetivos del Protocolo de Kioto $^{58}$. En el segundo ámbito, naturaleza y biodiversidad, el objetivo quedó fijado en proteger y restaurar la estructura y el funcionamiento de los sistemas naturales, tanto en la UE como en el mundo. En lo que se refiere a la relación entre medio ambiente, salud y calidad de vida, se estableció el objetivo de alcanzar una calidad del medio ambiente que contribuya a garantizar la salud pública, reduciendo los niveles de contaminación. Finalmente, el cuarto ámbito, consistente en la gestión de los recursos naturales y de los residuos, conllevaba el objetivo de velar por que el consumo de los recursos renovables y no renovables no supere el umbral de lo soportable por el medio ambiente.

Con el fin de hacer frente a los retos que se plantean en materia de medio ambiente, el sexto PMA estableció la necesidad de superar el enfoque estrictamente legislativo y sustituirlo por otro estratégico. De este modo, se propusieron cinco ejes prioritarios de acción estratégica: mejorar la aplicación de la legislación en vigor; integrar el medio ambiente en otras políticas; colaborar con el mercado; implicar a los ciudadanos y modificar sus comportamientos; tener en cuenta el medio ambiente en las decisiones relativas al ordenamiento y gestión del territorio.

Ya en 2006 el Consejo aprobó las directrices para revisar la estrategia para el desarrollo sostenible y aprovechar la sinergias con la Estrategia de Lisboa ${ }^{59}$. Su objetivo general se puede resumir en la elaboración de medidas que permitan a la UE mejorar la calidad de vida de las generaciones actuales y futuras, mediante una gestión eficaz de los recursos, aprovechando el potencial de innovación tecnológica y social que ofrece la economía y garantizando la prosperidad, la protección del medio ambiente y la cohesión social ${ }^{60}$.

La revisión de la estrategia supuso la actualización de los principales retos,

\footnotetext{
${ }^{58}$ En el caso de la UE, el objetivo consistía en reducir en un $8 \%$ las emisiones de gases de efecto invernadero (GEI) para el período 2008-2012 con respecto a los niveles de 1990.

${ }^{59}$ Consejo de la UE, Revisión de la Estrategia de la UE para un desarrollo sostenible (EDS UE), 10117/06, Bruselas, 9 de junio de 2006.

60 Fernández De GatTA SÁnChez, D., "Política ambiental de la Unión Europea", en LóPEZ Ramón, F. (coord.), Observatorio de Políticas Ambientales 2007, CIEMAT. Madrid, 2007, p. 61.
} 
determinando un objetivo general para cada uno: limitar el cambio climático y sus efectos negativos en el medio ambiente; garantizar unos transportes sostenibles; fomentar patrones de consumo y producción sostenibles; mejorar la gestión de los recursos naturales y evitar su explotación excesiva; fomentar la salud pública frente a las amenazas sanitarias; fomentar el desarrollo sostenible en el mundo. Al mismo tiempo, la UE determinó que los Estados miembros debían elaborar sus propias estrategias nacionales de desarrollo sostenible, estableciendo la necesidad de revisarlas cada dos años.

Como se ve, entre los fundamentos que iban a determinar la política y la normativa europeas en materia medioambiental cobraba relevancia de primer orden el cambio climático y sus consecuencias en cuanto reto y la lucha contra el mismo como objetivo. Así, en enero de 2007 la Comisión concretó la estrategia europea de lucha contra el cambio climático61, al tiempo que dio a conocer su propuesta ante los principales retos que se planteaban en materia energética en Europa, como son la seguridad de abastecimiento y, precisamente, el cambio climático, lo que la vinculaba claramente con la protección medioambiental ${ }^{62}$. Ambas fueron adoptadas por el Consejo Europeo ${ }^{63}$ y definirían la reforma que tendría lugar en estos ámbitos con el Tratado de Lisboa, al consagrarse una política integrada de medio ambiente y energía ${ }^{64}$.

\section{EL TRATADO DE LISBOA Y EL SÉPTIMO PMA}

El 13 de diciembre de 2007 fue firmado el Tratado de Lisboa, que entró en vigor el 1 de diciembre de 2009, quedando la normativa fundamental europea compuesta por el TUE y el TFUE. El art. 3 TUE establece como objetivo de la

\footnotetext{
${ }^{61}$ Comisión Europea, Comunicación de 10 de enero de 2007, Limitar el calentamiento mundial a $2^{\circ} \mathrm{C}$ - Medidas necesarias hasta 2020 y después, COM (2007) 2 final, 2007.

62 Comisión Europea, Comunicación de 10 de enero de 2007, Una política energética para Europa, COM (2007) 1 final, 2007.

${ }^{63}$ Consejo Europeo de Bruselas de 8 y 9 de marzo de 2007 - Conclusiones de la Presidencia, 7224/1/07 REV1.

${ }^{64} \mathrm{Al}$ tomar en consideración las implicaciones ambientales en la producción y el consumo de energía, ya no cabía concebir "una política energética que no tenga en cuenta como parte esencial de la misma su sostenibilidad, en pie de igualdad-como mínimo-con los aspectos de la seguridad del suministro y la competitividad". LÓPEZ SAKO, M. J., "La política energética sostenible de la Unión Europea (1)”, Noticias de la Unión Europea, núm. 322, 2011, p. 81.
} 
UE el desarrollo sostenible ${ }^{65}$, al determinar que "obrará en pro del desarrollo sostenible de Europa", que se basará en tres elementos: un crecimiento económico equilibrado, una economía social de mercado y "un nivel elevado de protección y mejora de la calidad del medio ambiente". El desarrollo sostenible queda así consagrado como objetivo de la UE, superando definitivamente el ámbito de la política de medio ambiente. Lo mismo sucede con la obligación de aplicar de modo generalizado en todas las políticas los objetivos y principios medioambientales, pues el art. 11 TFUE recoge expresamente que "las exigencias de la protección del medio ambiente deberán integrarse en la definición y en la realización de las políticas y acciones de la Unión, en particular con objeto de fomentar el desarrollo sostenible".

EI TFUE sitúa ahora la regulación de la política medioambiental en el Título XX, compuesto por los artículos 191 a 193. El primero de ellos recoge los principios vertebradores de la política ambiental europea o, si se quiere, los principios generales del Derecho en esta materia ${ }^{66}$, así como los objetivos concretos de la política europea de medio ambiente: la conservación, la protección y la mejora de la calidad del medio ambiente; la protección de la salud de las personas; la utilización prudente y racional de los recursos naturales; el fomento de medidas a escala internacional destinadas a hacer frente a los problemas regionales 0 mundiales del medio ambiente y en particular a luchar contra el cambio climático. La necesidad específica de luchar contra el cambio climático es una adición realizada por el Tratado de Lisboa, reflejo de la relevancia que para la UE había adquirido dicha cuestión.

Según el número 2 del citado art. 191 TFUE, la UE debe lograr un nivel de protección medioambiental elevado, si bien debe tenerse presente la diversidad de situaciones existentes en las distintas regiones del territorio europeo. Como se ve, esta política trata de garantizar el derecho de todos los ciudadanos de la UE a disfrutar del mismo nivel de protección medioambiental y el derecho de las

\footnotetext{
${ }^{65}$ De modo que la UE es la única región del mundo donde dicho objetivo "es declarado principio constitucional". TORRE-SCHAUB, M., "L'apport du principe de développement durable au droit communautaire: gouvernance et citoyenneté écologique", Revue du Marché Commun et de l'Union Européenne, núm. 555, 2012, p. 89.

${ }^{66}$ LÓPEZ RAMÓN, F., "La formación del ordenamiento ambiental", Revista Ambienta, núm. 124, 2018, p. 33.
} 
empresas a operar en las mismas condiciones de competencia, pero respetando las distintas circunstancias de cada país tanto como sea posible. El mismo párrafo enumera los cuatro principios en los que se basa la política de medio ambiente: cautela, acción preventiva, corrección de los atentados al medio ambiente en la fuente misma y quien contamina paga ${ }^{67}$.

La integración de la protección medioambiental en otras políticas europeas es especialmente apreciable en lo que se refiere a la energía, lo que quedó reflejado en la introducción de la regulación independiente de esta materia. La misma queda recogida en el Título XXI del TFUE, es decir, contigua a la normativa medioambiental y su único precepto (art. 194.1 TFUE) refleja el nexo entre ambas políticas pues establece los objetivos de la política energética europea "atendiendo a la necesidad de preservar y mejorar el medio ambiente".

Además de garantizar el funcionamiento del mercado de la energía y la seguridad de abastecimiento, así como de fomentar la interconexión de las redes energéticas, se recoge como objetivo de la política europea de energía "fomentar la eficiencia energética y el ahorro energético así como el desarrollo de energías nuevas y renovables", objetivo directamente relacionado con la agenda climática $y$, por tanto, con la política medioambiental ${ }^{68}$. La existencia de una estrecha relación entre los ámbitos energético y medioambiental queda, así, evidenciado también en su vertiente normativa en el Derecho Originario de la UE ${ }^{69}$. Es más, cabe afirmar que en la UE se desarrolla una política integrada en materia de

\footnotetext{
${ }^{67}$ La Comisión identifica el significado de cada uno de ellos. El principio de cautela significa que en caso de que haya indicios claros de que existe un problema medioambiental incipiente, se tomen medidas cautelares incluso cuando no se posee confirmación científica completa. El principio de acción preventiva responde a la concepción de que la mejor política de protección del medio ambiente consiste en intentar evitar cualquier forma de contaminación o de deterioro del mismo, en lugar de reparar los efectos de una acción perjudicial después de que ésta haya tenido lugar y cuando los daños son inevitables. El principio de corrección de los atentados al medio ambiente en la fuente misma de su producción supone aplicar de manera inmediata la solución correspondiente, al objeto de neutralizar al máximo los efectos de los ataques y evitar una posible progresión incontrolable de los mismos. El principio de quien contamina paga requiere determinar las actuaciones degradantes del medio ambiente susceptibles de constituir hechos que den lugar a responsabilidad, así como articular una reglamentación que identifique las infracciones que dan lugar a los perjuicios que haya que reparar y que individualice a las personas a quienes la infracción sea imputable. Comisión Europea, Un medio ambiente de calidad. La contribución de la UE, Oficina de Publicaciones de la CE. Luxemburgo, 2006, p. 4.

68 GuSEV, A. S., "Climate Changes...", cit., p. 81.

${ }^{69}$ Sobre esta cuestión, vid. Moussis, N., EU Environment \& Energy Policies: Two Interwoven Legislative Areas, European Study Service. Rixensart (Bélgica), 2009.
} 
energía y medio ambiente, de modo que la relación e interdependencia entre ambos ámbitos "se ha traducido en una necesaria simbiosis regulatoria"70. Lo cierto es que la producción normativa europea ya estaba reflejando la estrecha relación entre la lucha contra el cambio climático y la energía, principalmente por medio del impulso a las energías renovables, medida completamente favorecedora de la protección medioambiental ${ }^{71}$.

El 3 de marzo de 2010 la Comisión hacía pública la Estrategia Europa 2020, una propuesta para salir de la crisis económica internacional que estalló en 2008 y preparar la economía de la UE de cara a la siguiente década por medio de un crecimiento inteligente, sostenible e integrado ${ }^{72}$. En lo que a la sostenibilidad se refiere, la estrategia impulsó una economía de bajo nivel de carbono, eficiente en términos de recursos y competitiva. Para ello, se establecieron tres significativos objetivos en materia de clima y energía, a lograr en 2020: reducción del $20 \%$ de las emisiones de gases de efecto invernadero (GEI), con respecto a los niveles de 1990; aumento del $20 \%$ de la proporción de energías renovables en el consumo total de energía; y disminución del consumo en un $20 \%$ logrando una mayor eficiencia energética.

La Estrategia Europa 2020 tuvo una influencia notoria en el Séptimo PMA, más aún tras constatar la Comisión que, aún habiendo avanzado notablemente en determinados ámbitos, el sexto PMA podía llegar a no desarrollar todas las medidas previstas. En efecto, la evaluación final del mismo presentaba logros y deficiencias $^{73}$, pudiendo mencionarse, a modo de ejemplo, los siguientes: a pesar del aumento de la protección de la superficie terrestre (por medio de la

\footnotetext{
70 LÓPEZ SAKO, M. J., "La política energética...”, cit., p. 81.

${ }^{71}$ El mayor ejemplo venía dado por el conocido como "Paquete energía y clima", un conjunto de actos legislativos aprobados por el PE y el Consejo el 23 de abril de 2009 (todos en DOCE L 140, de 5 de junio de 2009): Directiva 2009/28/CE relativa al fomento del uso de energía procedente de fuentes renovables, Directiva 2009/29/CE por la que se perfecciona el comercio de derechos de emisión de GEI, Directiva 2009/31/CE sobre el almacenamiento geológico del dióxido de carbono, Decisión 406/2009/CE sobre el esfuerzo de los Estados miembros para reducir sus emisiones de GEI a fin de cumplir los compromisos adquiridos hasta 2020.

${ }^{72}$ Comisión Europea, Comunicación de la Comisión de 3 de marzo de 2010 "Europa 2020. Una estrategia para un crecimiento inteligente, sostenible e integrador", COM (2010) 2020, 2010. En junio el Consejo Europeo aprobó la propuesta y la hizo suya. Consejo Europeo de Bruselas de 17 de junio de 2010 - Conclusiones, EUCO 13/10.

${ }^{73}$ Comisión Europea, comunicación de 31 de agosto de 2011, VI Programa de Medio Ambiente - Evaluación Final, COM (2011), 531 final.
} 
Red Natura 2000) y del medio marino, así como la disminución de la contaminación de ríos y lagos, el objetivo global consistente en detener la pérdida de biodiversidad antes de 2010 no había sido alcanzado; aunque se constató que el consumo de recursos ya no aumentaba al mismo ritmo que el crecimiento económico, lo cierto es que continuaba creciendo en términos absolutos y el volumen global de residuos generados en la UE, en el mejor de los casos, se había estabilizado; y, a pesar del indudable éxito de la consecución antes de tiempo de los objetivos definidos en el Protocolo de Kioto, las emisiones de GEI en el sector del transporte estaban aumentando.

En noviembre de 2013 la UE aprobaba el Séptimo PMA ${ }^{74}$. Por medio del mismo, se asumió la constatación de que ya no es posible seguir tratando al medio ambiente como algo ajeno a la economía, de modo que ésta debe ser transformada para obtener más valor con menos recursos y cambiar los modelos de consumo. Para ello, el programa "establece una estrategia medioambiental a largo plazo, lo suficientemente adaptable y flexible para dar respuesta a los múltiples retos (...) fijando el rumbo hacia una economía verde y competitiva que salvaguardará los recursos naturales y la salud de las generaciones presentes y futuras" 75 . Como se ve, el programa trata de reforzar el impulso de actuaciones integradas en diferentes políticas y sectores, entre las que la energía es uno de los ámbitos principales ${ }^{76}$.

El nuevo programa estableció nueve objetivos prioritarios. Los tres primeros constituyen propiamente los ámbitos temáticos del programa. El primero consiste en proteger, conservar y mejorar el capital natural de la UE, lo que supone reforzar la defensa de la biodiversidad, así como actuar en lo que se refiere a la protección del suelo y de los recursos forestales. El segundo, la transformación de la UE en una economía hipocarbónica y eficiente en el uso de los recursos,

\footnotetext{
74 Decisión 1386/2013/UE del PE y del Consejo, de 20 de noviembre de 2013, relativa al Programa General de Acción de la Unión en materia de Medio Ambiente hasta 2020 "Vivir bien, respetando los límites de nuestro planeta", DOUE L 354 .

${ }^{75}$ Comisión Europea, Un medio ambiente sano y sostenible para las generaciones futuras, Oficina de Publicaciones de la UE. Luxemburgo, 2013, p. 15.

${ }^{76}$ El Séptimo PMA apostaba "claramente, y con tintes revolucionarios, por una economía baja en emisiones de carbono (...) y con previsiones muy importantes en materia de recursos, energía, energías renovables, biodiversidad, eco-innovación, transportes, etc.". FERNÁNDEZ DE GATTA SÁNCHEZ, D., "El Séptimo Programa...", cit., p. 93.
} 
requiere ante todo la aplicación de las medidas para alcanzar los objetivos en materia de clima y energía, así como transformar los residuos en recursos, apostando por la economía circular. El tercero consiste en la protección de la salud de los ciudadanos de la UE, para lo que se impulsa la reducción de la contaminación atmosférica y acústica.

Los objetivos prioritarios cuarto a séptimo constituyen el marco instrumental para realizar los objetivos temáticos: maximizar los beneficios de la legislación medioambiental europea; mejorar la base de conocimientos e información de la política de medio ambiente; asegurar inversiones en materia de clima y medio ambiente; y reforzar la integración de las cuestiones medioambientales en otras áreas, como las políticas agrícola, pesquera, de transporte y, especialmente, de energía. Finalmente, los dos últimos son objetivos que tratan de responder a desafíos locales, regionales y mundiales: aumentar la sostenibilidad de las ciudades e impulsar iniciativas globales para hacer frente a los desafíos medioambientales y climáticos a escala mundial.

\section{LA NECESIDAD DE UNA MAYOR AMBICIÓN}

En octubre de 2014 el Consejo Europeo aprobó el "Marco de actuación en materia de clima y energía hasta el año 2030"77, por el que se establecían nuevos objetivos en materia de clima y energía, ahora para 2030. No obstante, con la adopción de los actos que conforman el Paquete energía limpia los objetivos fueron ampliados ${ }^{78}$. El objetivo propuesto por la Comisión de reducción de las

\footnotetext{
${ }^{77}$ Consejo Europeo de Bruselas de 23 y 24 de octubre de 2014 - Conclusiones de la Presidencia, EUCO 169/14. Adoptaba la propuesta de la Comisión recogida en la comunicación de 22 de enero de 2014, Un marco estratégico en materia de clima y energía para el período 2020-2030, COM (2014) 15 final.

${ }^{78}$ El paquete había sido presentado por la Comisión Europea, comunicación de 30 de noviembre de 2016, Energía limpia para todos los europeos, COM (2016) 860 final. Los actos fueron adoptados el PE y el Consejo entre mayo de 2018 y junio de 2019: el 30 de mayo de 2018, la Directiva (UE) 2018/844 relativa a la eficiencia energética de los edificios (DOUE L 156, de 19 de junio de 2018); el 11 de diciembre de 2018, el Reglamento (UE) 2018/1998 sobre la gobernanza de la Unión de la Energía y la Acción por el Clima, la Directiva (UE) 2018/2001 relativa al fomento de la energía procedente de fuentes renovables, y la Directiva (UE) 2018/2002 relativa a la eficiencia energética (DOUE L 328, de 21 de diciembre de 2018); el 5 de junio de 2019, el Reglamento (UE) 2019/943 relativo al mercado interior de la electricidad, la Directiva (UE) 2019/944 sobre normas comunes para el mercado interior de la electricidad, el Reglamento (UE) 2019/941 sobre la preparación frente a los riesgos en el sector de la electricidad y el
} 
emisiones de GEI por lo menos en el $40 \%$ con respecto a 1990 se mantuvo, pero gracias a la Directiva sobre energías renovables se aumentó al $32 \%$ la cuota de energía procedente de fuentes renovables a alcanzar en el consumo final bruto de energía de la UE, y la Directiva de eficiencia energética determinó que el aumento de la eficiencia energética fuese de un mínimo del 32,5\%.

Tan relevante para el logro de la sostenibilidad y consecuencia directa de los objetivos establecidos en el séptimo PMA va a ser un segundo paquete legislativo, derivado del plan de acción europeo para poner en marcha un modelo económico circular. Los objetivos del plan consisten en promover la reparabilidad, durabilidad, reciclabilidad y eficiencia energética de los productos; reducir el desperdicio de alimentos y los desechos marinos; luchar contra la obsolescencia programada; reutilización del agua; impulsar la reciclabilidad y la biodegradabilidad de los plásticos ${ }^{79}$.

Una vez en marcha los paquetes legislativos referidos, la Comisión hizo pública a finales de 2018 su propuesta para desarrollar la transición a la energía limpia y para que las emisiones de GEI desaparezcan en Europa para $2050^{80}$, que se plasma en el objetivo de reducción de las emisiones entre el $80 \%$ y el $100 \%$. Se pretende que la UE deje de lado los combustibles fósiles en 2050, logrando así una situación de neutralidad climática, al tiempo que refuerza de cara al futuro la práctica europea de incorporar el objetivo de desarrollo sostenible a todos los ámbitos, razón por la que "la visión de la Comisión para un futuro climáticamente

Reglamento (UE) 2019/942 por el que se crea la Agencia de la UE para la Cooperación de los Reguladores de la Energía (DOUE L 328, de 14 de junio de 2019).

79 El plan había sido presentado por la Comisión Europea, comunicación de 2 de diciembre de 2015, Cerrar el círculo: un plan de acción de la UE para la economía circular, COM (2015) 614 final. Los actos también fueron adoptados el PE y el Consejo entre mayo de 2018 y junio de 2019: el 30 de mayo de 2018, la Directiva (UE) 2018/851 sobre residuos, la Directiva (UE) 2018/850 relativa al vertido de residuos, la Directiva (UE) 2018/852 relativa a los envases y residuos de envases y la Directiva (UE) 2018/849 relativa a los vehículos al final de su vida útil, a las pilas y a sus residuos, y a los residuos de aparatos eléctricos y electrónicos (DOUE L 150, de 14 de junio de 2018); el 5 de junio de 2019, la Directiva (UE) 2019/904 relativa a la reducción del impacto de determinados productos de plástico en el medio ambiente (DOUE L 155, de 12 de junio de 2019); y el 6 de junio de 2019, el Reglamento (UE) 2009/1009 sobre la puesta en el mercado de productos fertilizantes (DOUE L 170, de 25 de junio de 2019).

${ }^{80}$ Comisión Europea, comunicación de 28 de noviembre de 2018, Un planeta limpio para todos. La visión estratégica europea a largo plazo de una economía, próspera, moderna, competitiva y climáticamente neutra, COM (2018) 773 final. 
neutro abarca prácticamente todas las políticas de la UE"81.

Sin embargo, la realidad muestra que el grado de cumplimiento de los objetivos medioambientales y climáticos se está ralentizando desde 2014 debido a la recuperación económica tras la crisis de 2008. La propia Comisión advertía de ello en lo que se refiere a los compromisos en materia de clima y energía ${ }^{82}$, por lo que incidía en la necesidad de intensificar los esfuerzos para aumentar la eficiencia energética e impulsar las energías renovables. Los datos más recientes que pueden ser consultados confirman una buena tendencia general, si bien al mismo tiempo confirman las dudas planteadas por la Comisión ${ }^{83}$. El mejor dato es el referido a la reducción de emisiones de GEI, dado que la UE las había reducido para 2019 el $28 \%$ con respecto a los niveles de 1990 (25,9\% para la UE-27 sin Reino Unido). También es bueno el dato del porcentaje de energías renovables en el consumo total de energía, que en 2019 era del 18,9 \% (aún mejor para la UE-27, que alcanza el 19,7\%), si bien se aprecia la ralentización desde 2014. Ello es aún más acusado en el caso de la eficiencia energética, objetivo prácticamente logrado en 2014 pero que el aumento de consumo desde entonces dificultaba su logro en 2020.

Esa doble percepción, de estar en el buen camino, pero con un ritmo menor del necesario, se aprecia en otros datos como, por ejemplo, los referidos a la defensa del medio ambiente y la biodiversidad. Por un lado, el aumento de superficie protegida en la UE por aplicación del programa Natura 2000 es relevante, llegando al $18 \%$ de la superficie terrestre y doblándose la superficie marina protegida en la última década. Por el contrario, llama la atención que el

\footnotetext{
${ }^{81}$ El objetivo es una transición hacia un planeta limpio que "sea socialmente justa y refuerce la competitividad de la economía (...) garantizando empleos de alta calidad y un crecimiento sostenible en Europa, permitiendo simultáneamente ayudar a resolver otros retos medioambientales, como la calidad del aire o la pérdida de biodiversidad". Comisión Europea, comunicado de prensa de 28 de noviembre de 2018, La Comisión aboga por una Europa climáticamente neutra de aquí a 2050, IP/18/6543.

82 Comisión Europea, documentos de 9 de abril de 2019: Cuarto Informe sobre el estado de la Unión de la Energía, COM (2019) 175 final; Evaluación de 2018, con arreglo al artículo 24, apartado 3, de la Directiva 2012/27/UE relativa a la eficiencia energética, de los avances realizados por los Estados miembros en la consecución de los objetivos nacionales de eficiencia energética para 2020, COM (2019) 224 final; Informe de situación en materia de energías renovables, COM (2019) 225 final.

83 Datos obtenidos de Eurostat: ec.europa.eu/eurostat/web/climate-change/data/database (Greenhouse gas emissions) y ec.europa.eu/eurostat/web/energy/data/main-tables (Sustainable Development indicators). Última consulta, 20 de julio de 2021.
} 
gasto en protección medioambiental con respecto al PIB haya disminuido, puesto que, aún habiendo de tenerse presente el contexto de crisis económica entre 2008 y 2014, la recuperación desde entonces hace difícil de entender que el dato en cuestión haya pasado del $2 \%$ en 2010 al 1,9\% en $2019^{84}$.

La misma idea se puede apreciar en la evaluación del VII PMA que la Comisión hizo pública en mayo de 201985. Se destacó positivamente que el programa ha facilitado una importante transformación en la formulación de las políticas, la de medio ambiente pero también las demás, que deben integrar los objetivos ambientales; y ello ha posibilitado un mayor reconocimiento con respecto al hecho de que la protección ambiental, los beneficios sociales y el crecimiento económico sostenible van de la mano. La Comisión consideró que el programa ha mejorado la eficacia de la política medioambiental, que los objetivos prioritarios se están consiguiendo y que la visión para 2050 que contiene el programa sigue siendo válida pero, y aquí está la segunda vertiente, advertía de que se necesitarán ajustes a medida que surjan nuevos desafíos.

El informe de la AEMA sobre el estado y perspectivas 2020 reflejaba de un modo más acusado esa doble percepción ${ }^{86}$. Por un lado, resulta evidente que las políticas europeas en materia de medio ambiente, clima y energía, así como en todas las demás en lo que se refiere a la asunción y aplicación de los objetivos medioambientales, han supuesto relevantes avances en la protección medioambiental y la lucha contra el cambio climático. En cambio, por otro lado, el informe alerta de la grave situación de los ecosistemas europeos y de las amenazas ambientales que les acosan. En este sentido subraya la idea ya mencionada de que no será posible lograr el cumplimiento de todos los objetivos previstos para 2030 y 2050 con el actual ritmo de progreso, debiendo atenderse especialmente a ámbitos como la biodiversidad, uso de los recursos, impacto del cambio climático y los riesgos para la salud. Precisamente, las propuestas de

${ }^{84}$ Datos obtenidos de Eurostat: ec.europa.eu/eurostat/web/environment/biodiversity. Última consulta, 20 de julio de 2021.

${ }^{85}$ Comisión Europea, informe de 15 de mayo de 2019, Sobre la evaluación del VII Programa de Acción en materia de Medio Ambiente, COM (2019) 233 final.

${ }^{86}$ Agencia Europea del Medio Ambiente (2019): The European Environment. State and Outlook 2020. OPOCE, Luxemburgo. Recuperado de https://www.eea.europa.eu/publications/soer-2020 
mejora recogidas en el informe fueron incluidas en el contenido del Pacto Verde Europeo que presentaría poco después la Comisión.

\section{EL PACTO VERDE EUROPEO Y EL OCTAVO PMA}

La propuesta de Pacto Verde Europeo (PVE) fue presentada por la Comisión en diciembre de $2019^{87}$. Se trata de una hoja de ruta para hacer que la economía de la UE sea sostenible, transformando los retos en materia de clima y medio ambiente en oportunidades, y que ha sido considerado "el proyecto de política medioambiental más ambicioso de la historia europea"88. EI PVE es la estrategia para lograr una UE climáticamente neutra en 2050, si bien su proceso de implementación "auspicia un cambio sistémico en la economía y sociedad europea, que va más allá de la acción por el clima, para enmarcarse en el contexto del desarrollo sostenible" ${ }^{89}$. El documento prevé actuaciones para impulsar el uso eficiente de los recursos mediante el paso a una economía limpia y circular, detener el cambio climático, revertir la pérdida de biodiversidad y reducir la contaminación ${ }^{90}$.

EI PVE incide en todos los sectores de la economía, especialmente en aquellos más contaminantes, como el transporte, la energía, la agricultura, la industria, la construcción, el sector textil y el químico. El PVE insiste en dos ideas fundamentales que ya venían subrayando los últimos PMA: por un lado, junto a la configuración de políticas transformadoras sigue siendo indispensable la integración de la sostenibilidad en todas las políticas de la UE; por otro, "Ios

${ }^{87}$ Comisión Europea, comunicación de 11 de diciembre de 2019, El Pacto Verde Europeo COM (2019) 640 final.

88 Fernández De GatTA SÁnCheZ, D., "El ambicioso Pacto Verde Europeo". Actualidad Jurídica Ambiental, núm. 101, 2020, p. 81.

89 PÉREZ De LAS HeRAS, B., "La Unión Europea en la transición hacia la neutralidad climática: retos y estrategias en la implementación del Acuerdo de París", Revista española de Derecho Internacional, vol. 72, núm. 2, 2020, p.127.

90 EI PVE "utiliza como pilar la idea de sostenibilidad y economía circular unidas a la transición justa (...) cuyos costes y beneficios se distribuyan equitativamente entre los distintos grupos sociales, las industrias y las regiones y entre las generaciones presentes y futuras". ÁLVAREZ CUESTA, H., "Transición justa y lucha contra el cambio climático en el Pacto Verde Europeo y en el Proyecto de Ley de Cambio Climático en España". IUSLabor, núm. 2, 2020, p. 80. 
estados miembros deben garantizar que la legislación y las políticas pertinentes para el despliegue del PVE se hagan cumplir y se apliquen de manera efectiva"91.

En materia de energía y clima el PVE refleja la necesaria mayor ambición para lograr la neutralidad climática en 2050. Por ello, recoge la propuesta de elevar, como mínimo, al 50 \% la reducción de las emisiones de GEI (con respecto a 1990), a concretar por medio de una Ley del Clima Europea. En segundo lugar, el PVE apuesta por acelerar el proceso de descarbonización del sistema energético, intensificando la implantación de fuentes de energía renovable así como incrementando el nivel de eficiencia energética. También vinculada a la acción climática, el PVE apuesta por la defensa de la biodiversidad, no en vano el calentamiento climático repercute en la pérdida de la misma. De este modo, propone continuar con la ampliación de las zonas protegidas por la red Natura 2000, recuperar los ecosistemas dañados e incluso aumentar la biodiversidad en las áreas urbanas, haciendo más ecológicas las ciudades europeas. Siguiendo lo establecido en el PVE, el Consejo Europeo hizo suyo el objetivo de alcanzar una UE climáticamente neutra para 2050, instando a poner en marcha los trabajos y las medidas legislativas necesarias para su logro ${ }^{92}$.

A lo largo de 2020 se generalizó la situación de pandemia provocada por el Covid-19, al tiempo que en la UE lo hacía la idea referida a que las medidas a adoptar para superar la crisis económica derivada de dicho contexto debían seguir la vía propuesta en el PVE. En efecto, el PVE "tiene la oportunidad de incorporar las lecciones aprendidas de la pandemia del coronavirus, así como plantear respuestas estratégicas que contribuyan al cambio de modelo político, económico y social", para liderar así la transición ecológica en Europa ${ }^{93}$. En este sentido, "alinear los esfuerzos de recuperación pospandemia con unos objetivos climáticos ambiciosos podría fomentar transformadoras soluciones a largo plazo, que apunten a la construcción de un nuevo modelo de sociedad justa, sostenible

\footnotetext{
${ }^{91}$ Fernández De Gatta SÁncheZ, D., "Unión Europea: el ambicioso Pacto Verde Europeo y el futuro de la política ambiental de la Unión", en LóPEZ RAMÓN, F. (coord.), Observatorio de Políticas Ambientales 2020, CIEMAT. Madrid, 2020, p. 45.

92 Consejo Europeo de Bruselas de 12 de diciembre de 2019 - Conclusiones de la Presidencia, EUCO 29/19.

${ }^{93}$ MONGE, C., "El Pacto Verde Europeo es la luz al final del túnel". Esglobal, 29 de abril de 2020, https://www.esglobal.org/el-pacto-verde-europeo-es-la-luz-al-final-del-tunel/
} 
y descarbonizada"94. Es cierto que la implementación del PVE "dependerá en parte del comportamiento del PIB, de las tasas de desempleo y los niveles de déficit y deuda de los Estados miembros", pero la apuesta por su desarrollo brinda "la oportunidad de sentar las bases para la transformación del modelo de desarrollo de la UE"95.

La Comisión presentó en 2020 diferentes propuestas para desarrollar el contenido del PVE. Destacamos en primer lugar la conocida como Ley del Clima Europea ${ }^{96}$, es decir, el acto legislativo fundamental para garantizar la neutralidad climática de aquí a 2050. Se trataba de una propuesta de reglamento por el que la UE fija su objetivo de reducción de emisiones de GEI para 2030 en un mínimo del $50 \%$, preferiblemente del $55 \%$. En apoyo a esta propuesta, la Comisión presentó un nuevo modelo de industria para Europa que, precisamente, facilite el camino hacia la neutralidad climática al reducir su huella de carbono, volviéndose más ecológica y circular al tiempo que más competitiva ${ }^{97}$. En tercer lugar, la Estrategia sobre la Biodiversidad para $2030^{98}$, presentada con el fin de encaminar Europa hacia la recuperación ecológica y por la que se pretende que para 2030 sean zonas protegidas el $30 \%$ del suelo y de los mares de Europa. Finalmente, la Estrategia para la Integración del Sistema Energético ${ }^{99}$, con el objetivo de alcanzar un sistema energético europeo integrado, basado en una cuota cada vez mayor de energías renovables distribuidas geográficamente, con un uso más eficiente de los recursos que evite la contaminación y la pérdida de biodiversidad.

\footnotetext{
94 VILLAVICENCIO CALZADILLA, P., "La pandemia de Covid-19 y la crisis climática: dos emergencias convergentes". Revista catalana de Dret Ambiental, vol. XI, núm. 2, 2020, p. 19.

${ }^{95}$ LÁzaro Touza, L., Escribano Francés, G. y Crespi De Valldaura, V., "Transición energética y gobernanza climática en tiempos de pandemia". ICE Revista de Economía, núm. 916, 2020, p. 171.

${ }^{96}$ Comisión Europea, documento de 4 de marzo de 2020, Propuesta de Reglamento por el que se establece el marco para lograr la neutralidad climática ("Ley del Clima Europea"), COM (2020) 80 final.

${ }^{97}$ Comisión Europea, comunicación de 10 de marzo de 2020, Un nuevo modelo de industria para Europa COM (2020) 102 final.

98 Comisión Europea, comunicación de 20 de mayo de 2020, Estrategia de la UE sobre la biodiversidad de aquí a 2030 "Reintegrar la naturaleza en nuestras vidas", COM (2020) 380 final.

99 Comisión Europea, comunicación de 8 de julio de 2020, Impulsar una economía climáticamente neutra: Estrategia de la UE para la Integración del Sistema Energético, COM (2020) 299 final.
} 
En diciembre de 2020 el Consejo Europeo aprobó el objetivo de reducción de las emisiones GEI para 2030, que aumenta del $40 \%$ al $55 \%{ }^{100}$. Se trata de un objetivo vinculante para la UE que el Consejo Europeo instó a que se reflejase en la Ley del Clima Europea. Coincide esta institución con la Comisión en que la intensificación de la ambición climática europea tiene por objetivo impulsar el crecimiento económico sostenible, creando empleo, generando beneficios en materia de salud y medio ambiente, y contribuyendo a la competitividad europea en tecnologías ecológicas. Finalmente, en junio de 2021 el PE y el Consejo aprobaron el reglamento que recoge la normativa para el logro de la neutralidad climática ${ }^{101}$, consagrando así la ampliación del tercero de los objetivos en materia de clima y energía que no lo había hecho aún.

Meses antes la Comisión había presentado su propuesta en torno al octavo PMA $^{102}$, estrechamente vinculada al PVE y que establece los objetivos concretos y las condiciones necesarias para progresar en los avances que la UE viene realizando en materia de desarrollo sostenible ${ }^{103}$. El documento es la base a partir de la que el Consejo y el PE iniciaron las negociaciones correspondientes para definir el acto legislativo que lo apruebe ${ }^{104}$.

El octavo PMA tiene por objeto acelerar la transición ecológica de manera justa e inclusiva. El nuevo programa propuesto establece seis objetivos prioritarios temáticos, destacando en primer lugar la reducción irreversible y progresiva de las emisiones de GEl y el incremento de las absorciones por sumideros naturales o de otro tipo con el fin específico de lograr el objetivo para 2030 (reducción del

\footnotetext{
100 Consejo Europeo de Bruselas de 10 y 11 de diciembre de 2020 - Conclusiones de la Presidencia, EUCO 22/20.

101 Reglamento (UE) 2021/1119 del Parlamento Europeo y del Consejo, de 30 de junio de 2021, por el que se establece el marco para lograr la neutralidad climática y se modifica el Reglamento (CE) 401/2009 y (UE) 2018/1999 (“Legislación europea sobre el clima”) (DOUE L 243, de 9 de julio de 2021).

102 Comisión Europea, documento de 14 de octubre de 2020, Propuesta de Decisión del Parlamento Europeo y del Consejo relativa al Programa General de Acción de la Unión en materia de Medio Ambiente hasta 2030, COM (2020) 652 final.

${ }^{103}$ FeRnÁNDEZ De GatTA SÁNCHEZ, D., "El Octavo Programa Ambiental de la Unión Europea: la propuesta de decisión de 14 de octubre de 2020”, La Ley Unión Europea, núm. 87, 2020, p. 1.

${ }^{104}$ EI PE aprobó la propuesta el 9 de julio de 2021, fijando así su posición. Desde el PE se pretende que el VIII PMA "se convierta en una herramienta de gobernanza que guíe la política medioambiental de la UE más allá del Pacto Verde Europeo". Servicio de Estudios del Parlamento Europeo, De un vistazo (Pleno julio de 2021): Octavo Programa de Acción en materia de Medio Ambiente, PE 690.692 - julio de 2021. Recuperado de europarl.europa.eu
} 
$55 \%)$ y la neutralidad climática para 2050. En segundo lugar, el aumento de la capacidad de adaptación y la reducción de vulnerabilidad al cambio climático. El tercer objetivo determina un aceleramiento hacia una economía circular, mediante la evolución hacia un modelo de crecimiento regenerativo que devuelva al planeta más de lo que toma de él, así como la disociación del crecimiento económico del uso de los recursos y la degradación ambiental. En cuarto lugar, la consecución del objetivo cero en materia de contaminación sin sustancias tóxicas en aire, agua y suelo. El quinto objetivo se centra en la biodiversidad, en su protección, conservación y recuperación, centrándose particularmente en el aire, el agua, el suelo, los bosques, los humedales y los ecosistemas marinos. Finalmente, otro objetivo que abarca necesariamente diferentes ámbitos y políticas como es el fomento de la sostenibilidad ambiental y la reducción de las principales presiones climáticas y medioambientales asociadas a la producción y el consumo, en particular en los ámbitos de la energía, la industria, la construcción, la movilidad y el sistema alimentario.

Si la relación del octavo PMA con el PVE y las propuestas que lo están desarrollando es evidente, lo es aún más su influencia en la futura legislación en la materia, no en vano el programa constituye la herramienta para fijar el camino del desarrollo normativo de la misma. Ello queda reflejado en el último paquete legislativo propuesto por la Comisión, conocido como Objetivo $55^{105}$ y que se ha hecho público en julio de 2021. Se trata de una propuesta para cumplir con el objetivo de reducir las emisiones de GEI en el $55 \%$ para 2030 y lograr la neutralidad climática en 2050, así como para "hacer realidad el Pacto Verde Europeo" 106 .

Para ello, por un lado, se recogen modificaciones en normas vigentes en materia de comercio de derechos de emisión o de emisiones de vehículos (todos los matriculados a partir de 2035 serán de cero emisiones), en las directivas sobre combustibles alternativos (generalización de puntos de recarga eléctrica y

\footnotetext{
105 Comisión Europea, comunicación de 14 de julio de 2021, 'Objetivo 55': cumplimiento del objetivo climático de la UE para 2030 en el camino hacia la neutralidad climática, COM (2021) 550 final.

106 Comisión Europea, comunicado de prensa de 14 de julio de 2021, Pacto Verde Europeo: la Comisión propone transformar la economía y la sociedad de la UE para alcanzar los objetivos climáticos, IP/21/3541.
} 
repostaje de hidrógeno) y sobre fiscalidad de la energía, así como en las directivas sobre energías renovables (ampliándose al $40 \%$ el objetivo de energía procedente de fuentes renovables) y sobre eficiencia energética (duplicando la obligación de ahorro energético anual para los Estados miembros). Por otro lado, se proponen nuevos actos sobre mecanismos de ajuste en frontera del carbono, un mecanismo social de acción climática, sobre combustibles sostenibles en transporte aéreo y marítimo, y una nueva estrategia forestal (para preservar la biodiversidad y especialmente los bosques, cuya extensión se quiere ampliar notablemente).

\section{CONCLUSIONES}

La normativa fundamental europea en materia de medio ambiente es un ejemplo de las virtudes que tiene disponer de un ordenamiento jurídico común, cuya aplicación facilita el logro de un objetivo también común como es el desarrollo sostenible.

En los inicios de las CCEE las características del mercado común exigieron el establecimiento de unas reglas básicas que evitasen que las normativas medioambientales nacionales generasen situaciones contrarias a la libre competencia. Ante la falta de una regulación contenida en los Tratados originarios, se recurrió a los PMA para ir configurando la política europea de medio ambiente, inicialmente vinculada al desarrollo del mercado común pero ya desde el tercer programa centrada en la protección medioambiental. En efecto, los dos primeros PMA sirvieron de basamento para iniciar el desarrollo de una política medioambiental europea y, así, partiendo de un objetivo general consistente en compatibilizar un uso racional de los recursos naturales con el desarrollo económico posibilitaron que el tercer PMA incidiese en la prevención.

A partir de ahí, han sido los Tratados modificativos los que han ido configurando la actual política medioambiental de la UE, sirviéndose las instituciones de los PMA para su desarrollo inicial y determinar así el camino a seguir por los actos legislativos correspondientes. Así ha sucedido desde la reforma operada por el AUE, por medio de la que el medio ambiente pasó a tener regulación como política específica de las CCEE, estableciendo además que la protección 
medioambiental sería un componente de todas las políticas europeas, idea que comenzó a desarrollar el cuarto PMA.

Por su parte, el Tratado de Maastricht incluyó entre los fines a lograr por la UE un crecimiento sostenible respetuoso con el medio ambiente, para lo que el quinto PMA ahondó en una perspectiva de protección medioambiental generalizada en todos los ámbitos de la UE. El Tratado de Ámsterdam confirmó el desarrollo sostenible como objetivo de la integración europea y no exclusivamente de la política medioambiental, mientras que el sexto PMA estableció las vías a seguir para hacer frente a retos como el cambio climático, la defensa de la biodiversidad o la economía circular, lo que requiere actuar en numerosos ámbitos de las diferentes políticas europeas.

Es evidente que los dos avances más relevantes en la materia han venido determinados por la vinculación del crecimiento económico a la protección medioambiental, estableciendo el desarrollo sostenible como objetivo fundamental de la UE, y por la integración en la definición y en la realización de las demás políticas y acciones europeas de dicho objetivo. En ello, los PMA han sido herramientas completamente válidas para concretar los objetivos a lograr a partir de los fundamentos establecidos en los Tratados, no sólo en la política medioambiental, sino para la integración de los objetivos de protección del medio ambiente en las demás políticas europeas.

La evolución de las directrices y los principios que determinan la normativa fundamental de la UE en materia medioambiental analizada en este trabajo, por medio de los Tratados y de los PMA, permite concluir que el concepto de crecimiento sostenible es un elemento clave y fundamental en la determinación de los planteamientos para adoptar las medidas que conducen al desarrollo económico equilibrado. Todo ello ha quedado reflejado en el derecho originario vigente, gracias a la reforma operada por el Tratado de Lisboa, que dejó configurada una política integrada de medio ambiente y energía a la que se une la acción climática y que determinó lo que sería el séptimo PMA.

De este modo, en la actualidad, junto a la protección y defensa de la biodiversidad, la actividad de la UE se centra en la reducción de emisiones contaminantes, el aumento de la presencia de las energías renovables en el consumo de energía y el logro de una mayor eficiencia energética, por cuanto 
que son las herramientas más eficaces en la lucha contra el cambio climático. El Pacto Verde Europeo es el colofón, al menos hasta ahora, de esa evolución, al fundamentar los esfuerzos para recuperar la senda del crecimiento en el cumplimiento de los objetivos climáticos y medioambientales, más aún ante la ralentización en el grado de realización de los mismos, siendo el octavo PMA la herramienta que marca el camino a seguir para completar la transición hacia la sostenibilidad.

\section{BIBLIOGRAFÍA}

Alberton, M., "Environmental Protection in the EU Member States: Changing Institutional Scenarios and Trends", L'Europe en formation, núm. 363, 2012, pp. 287-300.

Alonso García, E., El Derecho Ambiental de la Comunidad Europea, Fundación Universidad-Empresa. Madrid, 1993.

Álvarez Cuesta, H., "Transición justa y lucha contra el cambio climático en el Pacto Verde Europeo y en el Proyecto de Ley de Cambio Climático en España", IUSLabor, núm. 2, 2020, pp. 74-100.

Armengol y Butrón De Múgica, D. y Carrillo Vargas, M. C., "El Cuarto Programa de la CEE en materia de medio ambiente (1987/1992) y la política ambiental comunitaria", Estudios Regionales, núm. 26, 1990, pp. 201-208.

Cuenca García, E., Economía de la Unión Europea, Pearson-Prentice Hall. Madrid, 2007.

De Carvalho Leal, V., "El Medio Ambiente como objeto de protección jurídica en el ámbito comunitario: hacia un sistema de Responsabilidad Ambiental", Nuevo Derecho, vol. 4, núm. 4, 2009, pp. 11-35.

Fernández De GatTA SÁnCHEZ, D., "La política ambiental comunitaria: especial referencia a los programas de acción", Revista de Instituciones Europeas, vol. 12, núm. 3, 1985, pp. 723-753. 
Fernández De Gatta SÁnchez, D., "Política ambiental de la Unión Europea", en LÓPEZ RAMÓn, F. (coord.), Observatorio de Políticas Ambientales 2006, CIEMAT. Madrid, 2006, pp. 59-98.

Fernández De Gatta Sánchez, D., "Política ambiental de la Unión Europea", en LÓPEZ RAmÓn, F. (coord.), Observatorio de Políticas Ambientales 2007, CIEMAT. Madrid, 2007, pp. 93-122.

Fernández De GatTa SÁnchez, D., "El Séptimo Programa Ambiental de la Unión Europea, 2013-2020", Revista Aragonesa de Administración Pública, núm. 4142, 2013, pp. 71-121.

Fernández De GatTa SÁnCHeZ, D., "El Octavo Programa Ambiental de la Unión Europea: la propuesta de decisión de 14 de octubre de 2020 (1)", La Ley Unión Europea, núm. 87, 2020.

Fernández De Gatta Sánchez, D., "El ambicioso Pacto Verde Europeo". Actualidad Jurídica Ambiental, núm. 101, 2020, pp. 78-109.

Fernández De Gatta Sánchez, D., "Unión Europea: el ambicioso Pacto Verde Europeo y el futuro de la política ambiental de la Unión”, en LÓPEZ RAMÓN, F. (coord.), Observatorio de Políticas Ambientales 2020, CIEMAT. Madrid, 2020, pp. 41-86.

Girón Larrucea, J. A., La Comunidad Europea como Organización Internacional, Centro de Estudios Ramón Areces. Madrid, 1999.

Gusev, A. S., "Climate Change Issues in a Transatlantic Context", L'Europe en formation, núm. 360, 2011, pp. 79-91.

Hinojo RojAS, M., "La política en materia de medio ambiente de la UE en perspectiva histórica: de Roma a Niza", Revista de Estudios Europeos, núm. 72 , 2018, pp. 26-73.

lázaro touza, L., Escribano Francés, G. y Crespi de Valldaura, V., "Transición energética y gobernanza climática en tiempos de pandemia". ICE Revista de Economía, núm. 916, 2020, pp. 165-183.

López Ramón, F., "Caracteres del Derecho Comunitario Europeo Ambiental", Revista de Administración Pública, núm. 142, 1997, pp. 53-74. 
LÓPEZ RAMÓN, F., "La formación del ordenamiento ambiental", Revista Ambienta, núm. 124, 2018, pp. 26-39.

LÓPEZ SAKO, M. J., "La política energética sostenible de la Unión Europea (1)", Noticias de la Unión Europea, núm. 322, 2011, pp. 81-92.

MeYer, J-H. y Poncharal, B., "L'européanisation de la politique environnementale dans les années 1970", Vingtième Siècle. Revue d'histoire, núm. 113, 2012, pp. 117-126.

MODAFFERI, C., "La política medioambiental europea. Estudio sobre el desarrollo de la protección ambiental y papel decisivo de la Unión Europea", Cuadernos Cantabria Europa, núm. 15, 2016, pp. 49-68.

MONGE, C., "El Pacto Verde Europeo es la luz al final del túnel". Esglobal, 29 de abril de 2020, disponible en https://www.esglobal.org/el-pacto-verde-europeoes-la-luz-al-final-del-tunel/

Moussis, N., EU Environment \& Energy Policies: two interwoven legislative areas, European Study Service. Rixensart (Bélgica), 2009.

Muñoz De Bustillo, R. y Bonete, R., Introducción a la Unión Europea: un análisis desde la economía, Alianza. Madrid, 2002.

Nieto Solís, J. A., La Unión Europea. Una nueva etapa en la integración económica de Europa, Pirámide. Madrid, 2005.

Pérez De Las Heras, B., "La Unión Europea en la transición hacia la neutralidad climática: retos y estrategias en la implementación del Acuerdo de París", Revista española de Derecho Internacional, vol. 72, núm. 2, 2020, pp. 117-141.

Plaza Martín, C., Derecho Ambiental de la Unión Europea, Tirant lo Blanch. Valencia, 2005.

Popeanga, V., "Environmental action programs in the European Unión Evolution and Specific", Annals of the "Constantin Bránçusi" University of Tárgu Jiu, Letter and Social Science Series, núm. 3, 2013, pp. 24-33.

TORRE-SchaUB, M., "L'apport du principe de développement durable au droit communautaire: gouvernance et citoyenneté écologique", Revue du Marché Commun et de l'Union Européenne, núm. 555, 2012, pp. 84-92. 
TORRES UGENA, N., "La protección del medio ambiente en el Acta única Europea", Noticias de la CEE, núm. 54, 1989, pp. 93-106.

Villavicencio Calzadilla, P., "La pandemia de Covid-19 y la crisis climática: dos emergencias convergentes". Revista catalana de Dret Ambiental vol. XI, núm. 2, 2020, pp. 1-27.

Wood, D. M. y YesiladA, B. A., The Emerging European Union, Pearson Longman. Nueva York, 2006.

YÁbAR Sterling, A., "El desarrollo sostenible, principio y objetivo común de la sociedad y el mercado, en la UE de nuestros días", Foro. Revista de ciencias jurídicas y sociales, núm. 0, 2004, pp. 75-94. 